\title{
Configuration of electrical spinal cord stimulation through real-time processing of gait kinematics
}

\author{
Marco Capogrosso $\oplus^{1,2,12}$, Fabien B. Wagner, ${ }^{1,12}$, Jerome Gandar ${ }^{1}$, Eduardo Martin Moraud ${ }^{1,3}$, \\ Nikolaus Wenger ${ }^{4,5}$, Tomislav Milekovic $\oplus^{1}$, Polina Shkorbatova $\oplus^{1}$, Natalia Pavlova ${ }^{6}$, \\ Pavel Musienk ${ }^{6,7,8}$, Erwan Bezard $\oplus^{9,10}$, Jocelyne Bloch ${ }^{11}$ and Grégoire Courtine $\circledast^{1,11^{1} \star}$
}

Epidural electrical stimulation (EES) of the spinal cord and real-time processing of gait kinematics are powerful methods for the study of locomotion and the improvement of motor control after injury or in neurological disorders. Here, we describe equipment and surgical procedures that can be used to acquire chronic electromyographic (EMG) recordings from leg muscles and to implant targeted spinal cord stimulation systems that remain stable up to several months after implantation in rats and nonhuman primates. We also detail how to exploit these implants to configure electrical spinal cord stimulation policies that allow control over the degree of extension and flexion of each leg during locomotion. This protocol uses real-time processing of gait kinematics and locomotor performance, and can be configured within a few days. Once configured, stimulation bursts are delivered over specific spinal cord locations with precise timing that reproduces the natural spatiotemporal activation of motoneurons during locomotion. These protocols can also be easily adapted for the safe implantation of systems in the vicinity of the spinal cord and to conduct experiments involving realtime movement feedback and closed-loop controllers.

Introduction

Decades of research in physiology have demonstrated that sensorimotor circuits that produce muscle synergies are embedded in the mammalian spinal $\operatorname{cord}^{1,2}$. These circuits process sensory information arising from the moving limbs and descending inputs originating from various brain regions to produce adaptive motor behaviors. A spinal cord injury (SCI) interrupts the communication between the spinal cord and supraspinal centers, depriving these sensorimotor circuits of the sources of excitation and modulation that are necessary to produce movement.

A series of studies in animal models and humans have shown that electrical neuromodulation of the lumbar spinal cord using EES is capable of reactivating these circuits. Continuous EES restored locomotion in paralyzed rats and isolated leg movements in individuals with motor paralysis ${ }^{3-9}$.These studies have provided a proof of concept highlighting the potential of EES to transform SCI medicine. This has triggered a surge of interest in developing an evidence-based framework that can enable EES to be used for clinical applications. Computational models ${ }^{10-12}$ and experimental studies ${ }^{13,14}$ have provided evidence that EES recruits large sensory afferents, particularly proprioceptive feedback circuits $^{11}$. The recruitment of these pathways via electrical stimulation leads to the activation of motoneurons through monosynaptic and polysynaptic proprioceptive circuits. The excitatory drive produced by EES also increases the general excitability of the spinal cord, which enables residual descending inputs to trigger volitional movement ${ }^{3,15}$. In addition, the natural modulation of

${ }^{1}$ Center for Neuroprosthetics and Brain Mind Institute, School of Life Sciences, Swiss Federal Institute of Technology (EPFL), Lausanne, Switzerland. ${ }^{2}$ Platform of Translational Neuroscience and Department of Neuroscience and Movement Science, Faculty of Science and Medicine, University of Fribourg, Fribourg, Switzerland. ${ }^{3}$ Nuffield Department of Clinical Neurosciences, University of Oxford, Oxford, UK. ${ }^{4}$ Department of Neurology with Experimental Neurology, Charité Universitatsmedizin, Berlin, Germany. ${ }^{5}$ Center for Stroke Research Berlin, Charité-Universtitatsmedizin Berlin, Berlin, Germany. ${ }^{6}$ Institute of Translational Biomedicine, St. Petersburg State University, St. Petersburg, Russia. ${ }^{7}$ Russian Research Center of Radiology and Surgical Technologies, Healthcare of the Russian Federation, St. Petersburg, Russia. ${ }^{8}$ Saint-Petersburg State Research Institute of Phthisiopulmonology, Ministry of Healthcare of the Russian Federation, St. Petersburg, Russia. ${ }^{9}$ University of Bordeaux, Institut des Maladies Neurodégénératives, UMR 5293 Bordeaux, France. ${ }^{10} \mathrm{CNRS}$, Institut des Maladies Neurodégénératives, UMR 5293 Bordeaux, France. ${ }^{11}$ Department of Neurosurgery, University Hospital of Lausanne (CHUV), Lausanne, Switzerland. ${ }^{12}$ These authors contributed equally: Marco Capogrosso, Fabien B. Wagner.

*e-mail: gregoire.courtine@epfl.ch 
proprioceptive feedback circuits during movement execution contributes to steering the effects of EES toward functionally relevant pathways ${ }^{11}$. Due to the phase-dependent modulation of proprioceptive feedback circuits, the excitatory drive produced by the stimulation is directed to specific ensembles of leg motoneurons that match the phase of the movement ${ }^{11}$.

The efficacy of this mechanism relies on descending signals that tune the gain of sensorimotor feedback circuits based on ongoing task requirements ${ }^{16}$. However, the interruption of descending pathways after SCI substantially alters this task-dependent modulation of sensorimotor feedback circuits $^{17,18}$. Consequently, this mechanism is not sufficient during continuous EES to produce the robust alternation between extensor and flexor muscle synergies that is required for locomotion, particularly when descending pathways are severely damaged ${ }^{18}$. Moreover, continuous EES is intrinsically unfit to tune and control the modulation of specific motoneuron pools during specific phases of the gait cycle ${ }^{11}$. Such modulation is essential to addressing subject-specific deficits, such as left-right asymmetries or differential deficits of flexion or extension muscle synergies ${ }^{11,19,20}$. To overcome these limitations, we developed novel EES protocols termed 'spatiotemporal neuromodulation therapies, ${ }^{8,20}$.

During locomotion, the activation of motoneuron pools underlying extension and flexion muscle synergies emerge in specific segments of the spinal cord ${ }^{21}$. Indeed, locomotion is associated with motoneuron activation hot spots that migrate during the progression of the gait cycle ${ }^{22}$. As EES activates motoneurons through the modulation of proprioceptive feedback circuits that innervate distinct segments, spatially segregated motor pools may be accessed when delivering targeted stimulation of individual dorsal roots. In turn, the application of these spatially selective EES bursts with a temporal sequence that reproduces the natural activation of leg motoneurons during locomotion ${ }^{8,20}$ allowed a robust facilitation of extension and flexion muscle synergies in both rodent and nonhuman primate models of $\mathrm{SCI}^{8,20}$. This conceptual and technical framework is directly applicable to the development of spatiotemporal neuromodulation therapies for enabling leg motor control in humans with SCI.

The design and implementation of spatiotemporal neuromodulation therapies requires technologies capable of achieving the spatial and temporal specificities necessary to reproduce the natural pattern of spinal cord activation during locomotion (Fig. 1). These technologies include a spinal implant located over the epidural aspect of the spinal cord to deliver spatially selective stimulation protocols and a real-time control system that extracts information about actual or desired leg kinematics to adjust the temporal structure of the stimulation patterns.

To complement and expand our previous work ${ }^{8,11,20,23-26}$, here we present the detailed technical features and mechanisms underlying the design of targeted spinal implants, the methods for extracting key events of the gait cycle, the surgical procedures for implanting chronic systems for spinal cord stimulation and EMG recordings, and the step-by-step experimental protocols for the optimization of the spatial and temporal specificity of stimulation bursts. These protocols should support the implementation and configuration of spatiotemporal neuromodulation therapies in rodent and nonhuman primate models.

These protocols can be easily adapted for the safe and long-lasting implantation of recording or stimulation systems in the vicinity of the spinal cord, and to conduct behavioral experiments involving real-time monitoring of movement feedback and closed-loop gait controllers. Although this protocol is focused on applications in rats and nonhuman primates, it is also possible to tailor this approach for use in other animal models. In addition, this approach has the potential for eventual application in humans to enable movement and enhance motor recovery in patients with various neuromotor disorders.

\section{Choice of animal models}

In this protocol, we outline the procedures to implement and optimize spatiotemporal stimulation protocols in rat and monkey models of SCI. However, these protocols can be easily adapted to other animal models of neuromotor disorders, and even to clinical settings.

\section{Rat models of SCI}

Rats are the most commonly used animals in SCI research. Although several models of SCI exist ${ }^{27}$, the two most common involve surgical cuts and robotically controlled contusions. Surgical cuts of the spinal cord lead to reproducible SCIs. The cut can be either complete or incomplete, resulting in the precise interruption of specific neural pathways. Accordingly, the SCI can lead to complete or partial 


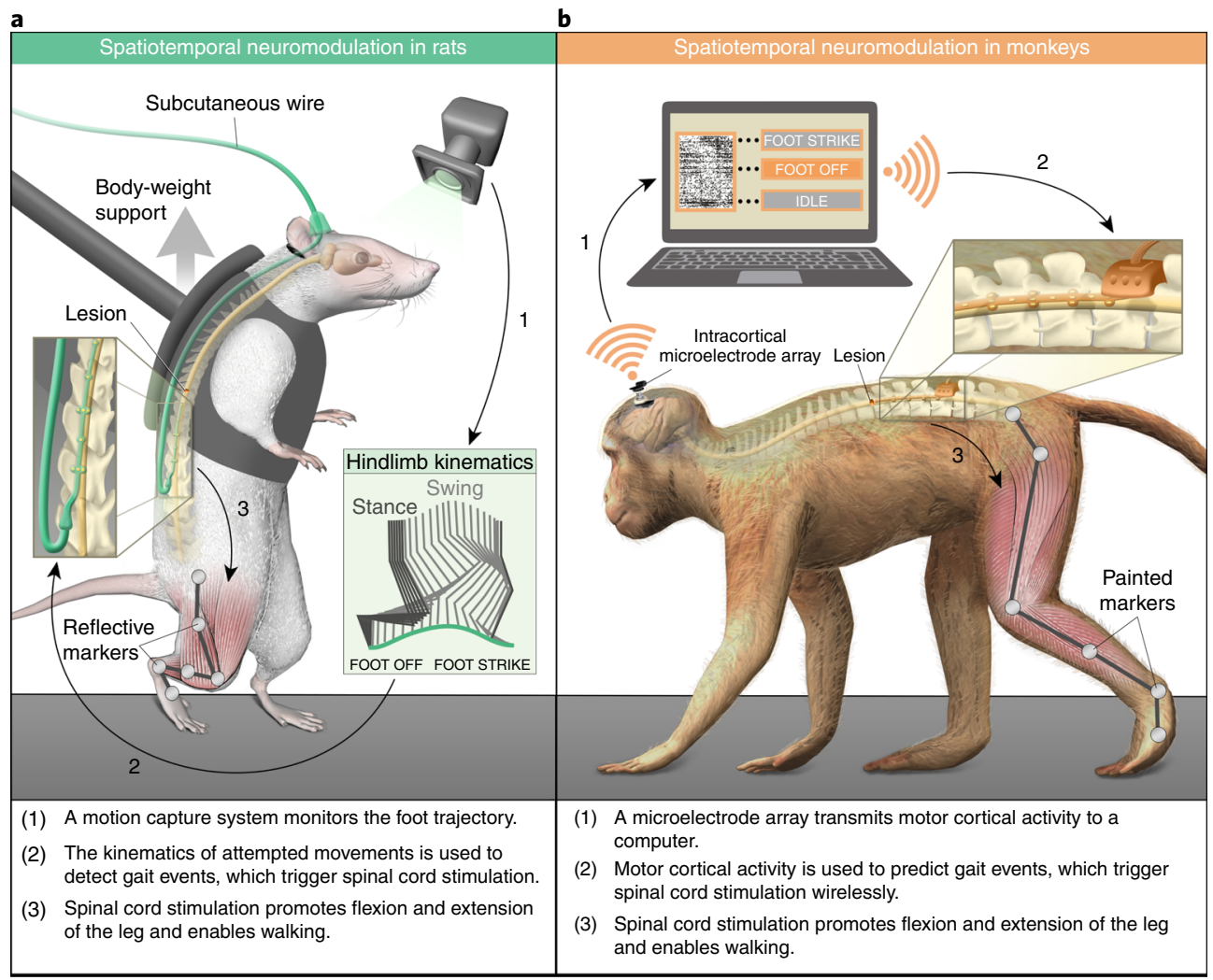

Fig. 1 | Conceptual and technological framework underlying spatiotemporal neuromodulation. a, (Rats) Kinematics of both legs are obtained using a camera-based motion-capture system monitoring the three-dimensional (3D) position of IR-reflective spherical markers placed over the main joints of the leg (1). A real-time algorithm detects the occurrence of gait events ('Foot Off' and 'Foot Strike' events), using the stereotypical shape of the trajectory described by the ankle over time (2). Spinal cord stimulation protocols promoting either flexion or extension of the leg are triggered upon detection of the 'Foot Off' and 'Foot Strike' gait events, respectively (3). The spinal implant is connected by subcutaneous wires to a connector placed on the head of the animal. This connector also gives access to electromyographic wires implanted in the main leg muscles. A robotic body-weight-support system assists the animal during bipedal locomotion. b. (Monkeys) Neuronal signals from the leg area of the motor cortex are extracted through intracortical microelectrode array recordings and broadcast wirelessly to an external computer (1). Neuronal signals are preprocessed and used by a real-time decoder that detects or anticipates the occurrence of the 'Foot Off' and 'Foot Strike' gait events (2). Spinal cord stimulation protocols promoting either flexion or extension of the leg are triggered upon detection of the 'Foot Off' and 'Foot Strike' gait events, respectively (3). The spinal implant is connected to an implantable pulse generator that communicates wirelessly with an external computer controlling the execution of the stimulation patterns. Markers are painted on each of the principal leg joints for 3D offline kinematic analysis using a specialized video-based motion tracking system.

paralysis ${ }^{28}$. For experiments involving locomotion, the transection is usually performed around the T8 vertebral level. This injury spares the lumbosacral circuits that control leg movement. When the transection is complete, the animal will lose control over its bladder. Manual voidance of the bladder is necessary twice per day, every day. Due to the absence of supraspinal inputs, complete transections are advantageous for studying and probing spinal circuits without confounding factors.

To produce complete motor paralysis without transecting all the fibers in the spinal cord, we recommend using a model of staggered hemisections ${ }^{3,29}$. In this model, two hemisections are performed at different vertebral levels, such as T7 and T10, on opposite sides of the spinal cord. Reorganization of circuits within the spared tissue bridge can support recovery ${ }^{3,29}$. This wellcontrolled and reproducible model is thus very attractive for the study of the mechanisms of recovery after severe SCI. Animal care procedures for this model are similar to those described for the complete transection model.

Other partial lesions include a lateral hemisection, a dorsal hemisection, and even a T-shaped cut. These injuries interrupt specific neural pathways, but because many alternative routes are spared, they are usually followed by an extensive spontaneous recovery that limits the heuristic value of these models for evaluating long-term recovery. During the first few weeks after injury, however, the 
clear-cut deficits allow study of the immediate impact of neurotechnologies to alleviate motor deficits. Due to extensive tissue sparing, autonomic functions are usually not impacted, which reduces animal care requirements and improves the animal's overall well-being.

The most clinically relevant models involve robotically controlled contusions of various severities ${ }^{20}$ or crushes made with tweezers. Although these models are well suited to the study of long-term recovery, the intrinsic variability of such injuries renders group comparisons and mechanistic studies more difficult. Typically, such experiments require a much larger number of animals to obtain statistically meaningful outcomes. Animal care requirements vary with the amount of damaged tissue, from intense care to near-normal procedures.

We have delivered spatiotemporal stimulation protocols in rats with complete transection, staggered hemisections, lateral hemisection and severe contusions ${ }^{3,20,29}$. Users of this protocol should select the most appropriate SCI model to use according to the scientific question that is being addressed.

\section{Primate models of SCI}

Intensive animal care procedures, ethical considerations and regulatory constraints pose boundaries on the type of injury models that can be implemented in nonhuman primates ${ }^{30}$. For testing the immediate facilitation of walking when delivering spatiotemporal neuromodulation, we adopted a model of lateralized SCI. This lesion consists of placing a cut on one side of the thoracic spinal cord to interrupt the dorsolateral column, wherein the corticospinal tract resides. This SCI leads to a temporary paralysis of the leg ipsilateral to the lesion but does not compromise the autonomic function and postural control. Animals exhibit prominent spontaneous recovery. They regain a near-normal gait $\sim 4-8$ weeks after the lesion ${ }^{31,32}$. After a complete lateral hemisection, functional recovery is slower and incomplete ${ }^{33}$, in particular after a cervical $\mathrm{SCI}^{34}$. Reproducible models of hemicontusion SCI have also been developed in nonhuman primates ${ }^{35}$. Models of complete transection are difficult to justify ethically.

\section{Spatial specificity and design of spinal implant Anatomical considerations}

The mammalian spinal cord is anatomically organized in a finite number of segments (Fig. 2). Spinal segments are identified anatomically as the regions receiving fibers from the homonymous spinal roots. The lumbosacral enlargement of rats and macaque monkeys contains a total of six and seven segments, respectively ${ }^{36}$. In both rats and monkeys, the majority of these segments are located under two or three vertebrae. Consequently, there is an offset between the anatomical position of each spinal segment and its corresponding vertebrae (Fig. 2). The dorsal and ventral roots enter the spinal cord from the foramina that are located caudally to the homonymous vertebrae (Fig. 2). Therefore, they run longitudinally along the spinal cord until they intersect their target spinal segments. As the dura mater maintains the spinal roots packed around the spinal tissue, they are immersed within the highly conductive cerebrospinal fluid (CSF) (Fig. 2). Due to these anatomical conditions, the spinal roots constitute a low-threshold target for electrical stimulation.

These considerations are particularly relevant for the dorsal roots ${ }^{10,12,37,38}$. Indeed, the electrodes of the spinal implant are located dorsally and thus in close apposition to these roots, which embed sensory afferent fibers (Fig. 2). When recruiting these fibers, the excitatory drive produced by the electrical stimulation first propagates toward the segments innervated by the root containing the recruited fibers, before spreading to other circuits receiving inputs from collaterals of these fibers ${ }^{10,11}$. Consequently, the accuracy in the recruitment of specific rootlets determines the ability to target a specific segment. In turn, the physiological spread of the stimulation-induced excitatory drive due to fibers' collaterals defines the upper-bounds of the potential for spatial specificity. Therefore, the optimization of spinal implants requires integrating not only the dimensions of spinal segments but also the path of the dorsal roots in the design of electrode locations ${ }^{8,20,39}$ (Fig. 2).

\section{Spinal implants}

Spinal implants should be custom manufactured for use in either rats or monkeys. Thin-film technologies ${ }^{8,20}$ and soft silicone interfaces ${ }^{25,40}$ have been used for this purpose. The surface area of the electrode active sites is determined by the maximum charge injection capacity, given the range of stimulation parameters used in each species. In rats, the commonly used stimulation currents range from 50 to $500 \mu \mathrm{A}$, and the optimal pulse width is typically set to $200 \mu \mathrm{s}^{10,20}$. In monkeys, these 

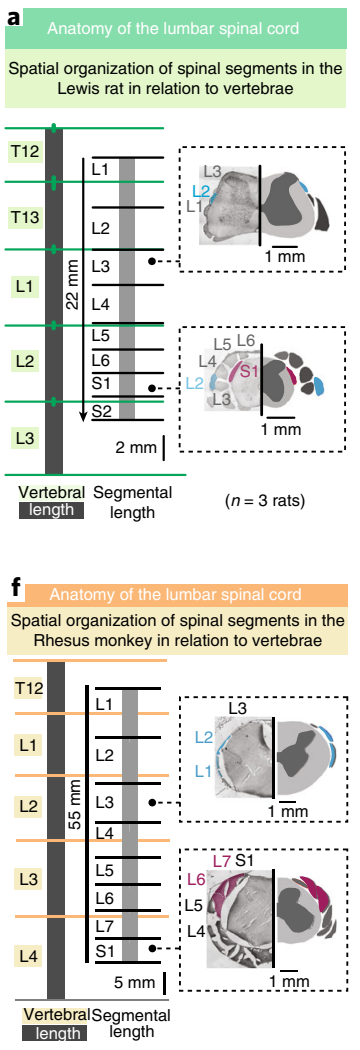
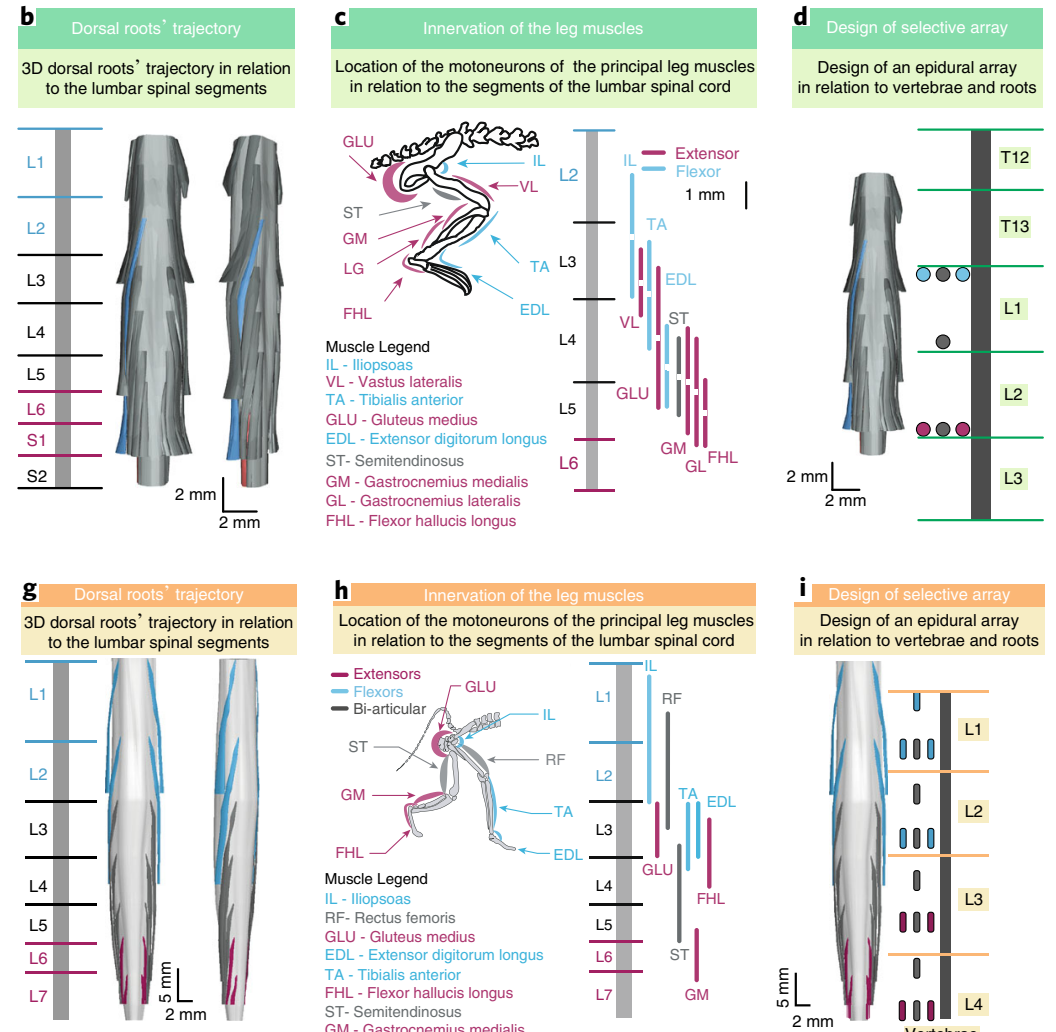

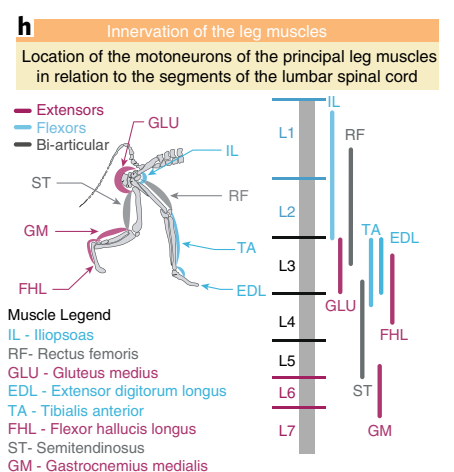

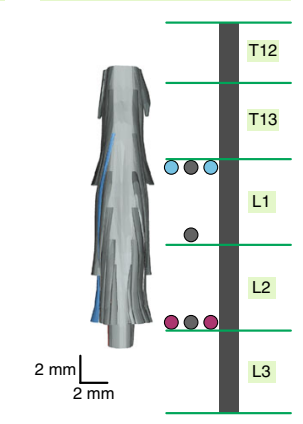

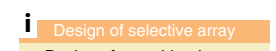

Design of an epidural array
in relation to vertebrae and roots

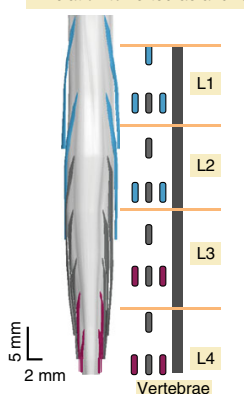

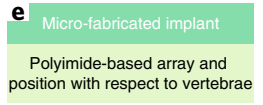
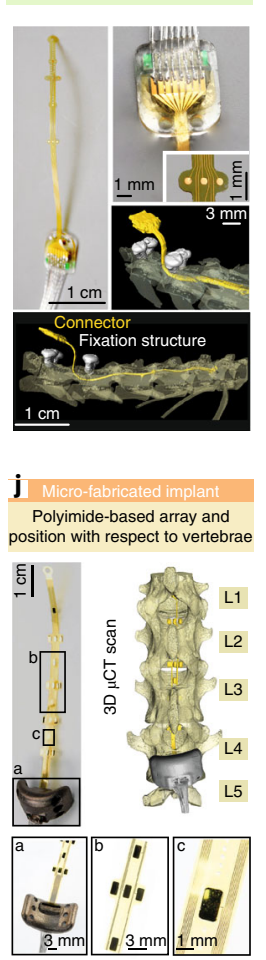

Fig. 2 | Spatially selective spinal implants tailored to the anatomy of the spinal cord. a-j, Anatomical organization of the lumbar spinal cord and tailored design of spinal implants in rats (a-e) and Rhesus monkeys (f-j). $\mathbf{a}, \mathbf{f}$, The anatomy of the lumbar spinal cord. On the left, average position of the vertebrae covering the lumbosacral spinal cord in rats (a, green) and Rhesus monkeys (f, yellow). The insets show coronal sections of the cord at the L3 and S1 spinal segments (left, photographs; right, digital reconstructions of the dorsal roots, and white and gray matter; cyan, the two most rostral dorsal roots; magenta, the two most caudal dorsal roots). $\mathbf{b}, \mathbf{g}$, 3D reconstruction of the dorsal root trajectory relative to the spinal segments. Frontal and lateral views of the cord are shown with the color-coding convention in a. c,h, Innervation of the main leg muscles with respect to the spinal segments (cyan, flexor muscles; magenta, extensor muscles; gray, bi-articular muscles). This information is critical to achieving specificity. Spinal implants are tailored to target the roots that innervate these muscle groups. $\mathbf{d}, \mathbf{i}$, The ideal location of the spinal implant with respect to the dorsal roots and vertebrae (cyan, electrodes that potentially target the flexor muscles; magenta, electrodes that potentially target the extensor muscles). The surgical implantation procedure used for the spinal implant is based on this information. $\mathbf{e}, \mathbf{j}$, Photographs depicting the microfabricated spinal implants and the associated 3D microCT scans of the implant after implantation. a,b,d adapted with permission from Wenger et al. ${ }^{20}$, Springer Nature Limited; f-j adapted with permission from Capogrosso et al. ${ }^{8}$, Springer Nature Limited. Photographs, copyright 2016 J. Laurens. All animal experiments depicted in this figure were approved by the local authorities.

currents typically vary between 1 and $5 \mathrm{~mA}$ (or $1-10 \mathrm{~V}$, for implants with impedances $<2 \mathrm{k} \Omega$ ), with a pulse width of $380 \mu$ s (ref. ${ }^{8}$ ). However, to maintain a sufficient margin, the implant should be designed with features that allow the electrodes to sustain twice the maximum planned charge injection. When designing new types of electrodes, it is also important to ensure that the charge injection remains below the limit of water electrolysis, which could lead to adverse effects on the neighboring tissues. The charge injection limit depends on the material and size of the electrodes, as well as the various stimulation parameters such as amplitude, frequency, pulse width and shape of the stimulation pulse $\mathrm{s}^{41,42}$.

For safety reasons, it is important to consider twice the maximum planned charge injection when designing the implant. The sizes of the active sites and conductive tracks linking them to the interface connector determine the electrical impedance of the electrode. To design spinal implants, we suggest using active sites with an impedance of $<50 \mathrm{k} \Omega$ for rats (corresponding to an electrode diameter of $300 \mu \mathrm{m}$ ) and $<5 \mathrm{k} \Omega$ for monkeys (corresponding to electrodes of $0.5 \times 1 \mathrm{~mm}$, for example). Note that the impedance of the electrodes determines the compatibility of the interface with commercially available stimulation systems that are bounded to voltage and current limits within certain impedance ranges. From our experience, the impedance of the electrodes and current thresholds for eliciting motor responses remain stable over time. Moreover, we have not observed any substantial inflammatory response for up to 6 weeks after implantation in rats ${ }^{20,25,26}$. In humans, epidural electrodes 
routinely used for the treatment of chronic pain can show variations in impedance during the initial 6-8 weeks after implantation, due to the formation of a fibrotic layer. However, impedance values and stimulation parameters remain relatively stable in the long term ${ }^{43,44}$.

To deliver current through the spinal implant, a highly reliable and resistant interface must be established between the relatively soft material constituting the implant and a cabling system connecting the track of each active site to a wire. This interface represents the most delicate component of the system. To avoid breakage, especially with thin-film technologies ${ }^{8,20}$, we designed a bone orthosis to host the interface. In rats, this orthosis can be built during implantation using dental cement. In monkeys, the orthosis consists of a titanium anchor system that is positioned between the L4 and L5 vertebrae and screwed onto the L5 vertebra (Fig. 2). Both solutions ensure a robust encapsulation of the fragile electrode-wire interface that can resist long-term chronic implantation. We did not observe neurological complications due to long-term implantation of these orthoses. However, it is important to cover the orthoses with muscles and fascia to prevent the orthosis from puncturing the skin.

\section{Setup for electrical stimulation}

In rats, electrical stimulation can be delivered by an external multichannel stimulator (e.g., an IZ2H stimulator) controlled by a computer or real-time processor (e.g., an RZ2 BioAmp Processor) that is connected to the spinal implant through wired connections. In monkeys, behavioral tasks require the animal to behave freely without any tethered connections or cumbersome electronics. This application requires a wireless pulse generator with real-time triggering capacities that may be mounted on the head through a pedestal or surgically implanted inside the animal's chest or abdomen. Off-the-shelf head-mounted solutions are not powerful enough to deliver sufficient current (up to $10 \mathrm{~mA}$ ) for EES applications. As of today, the only solution available for research applications consists of medical-grade implantable pulse generators (IPGs) that are routinely used to deliver EES or deep-brain stimulation to alleviate chronic pain or Parkinson's disease symptoms. However, the use of such IPGs (e.g., the Activa RC manufactured by Medtronic) requires customized solutions for supporting wireless and real-time communication with the IPG. In our applications, we collaborated with Medtronic to develop a telemetry system consisting of an antenna linked to an interface worn by the animal and placed in a custom-made jacket ${ }^{8}$. This interface transmits information wirelessly via a Bluetooth connection to an external computer. A software interface provides the opportunity to change the parameters of stimulation (amplitude, frequency, pulse width, timing), to select electrode configurations and to trigger the onset and end of stimulation protocols.

\section{Evaluation of the spatial selectivity of spinal implants}

Electrophysiological recordings should be conducted intraoperatively to verify the spatial selectivity of the spinal implant and fine-tune its anatomical position (Figs. 3-5). Application of a single current pulse (0.5 ms pulse width) at 1.2-1.4 times motor threshold through the selected electrode contact will elicit motor responses in leg muscles that can be recorded with bipolar electrodes inserted into the muscles or placed over their surface. The motor responses involve monosynaptic and polysynaptic components that correspond to the recruitment of proprioceptive feedback circuits ${ }^{8,13,45}$, also termed 'spinal reflexes', and a direct response elicited by the recruitment of motor nerves. The monosynaptic component of these responses appears at the lowest threshold because they result from the direct activation of Ia-afferent fibers ${ }^{10}$, which have the largest diameter. These fibers establish excitatory synaptic connections to all the motoneurons innervating their homonymous muscle ${ }^{11,46}$. Because the location of motoneuron pools along the rostrocaudal extent of the spinal cord is known (Fig. 2), the relative activation of each spinal segment (or dorsal root) may be inferred from the normalized activation of each leg muscle ${ }^{8}$ (Fig. 5). It is important to understand that spatiotemporal neuromodulation does not seek to activate single muscles, but rather to modulate synergistic muscle groups located in a given segment. However, the anatomical locations of antagonist muscles may partly overlap. For example, a subset of motoneurons innervating the tibialis anterior (TA) and gastrocnemius medialis (GM) are located in lower lumbar segments, which may limit the specificity of stimulation protocols during static conditions. However, the sensory information and interactions with residual descending inputs gate the stimulation effects toward functionally relevant muscles during the given phase of the movement, which improves the specificity of the stimulation ${ }^{11,47,48}$. The spatial selectivity of EES thus increases during movement execution as compared with static measurements. During the implantation procedure, the most time-saving strategy consists of optimizing 

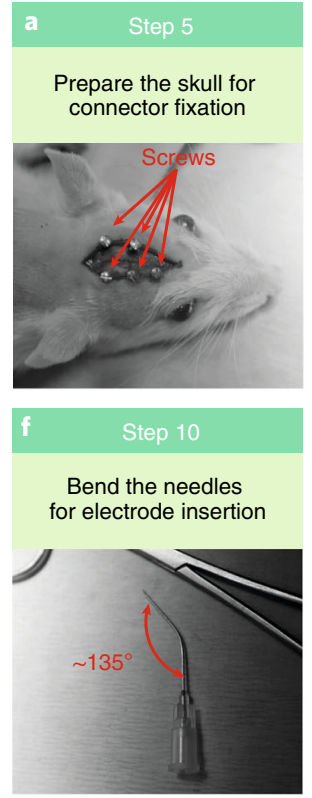
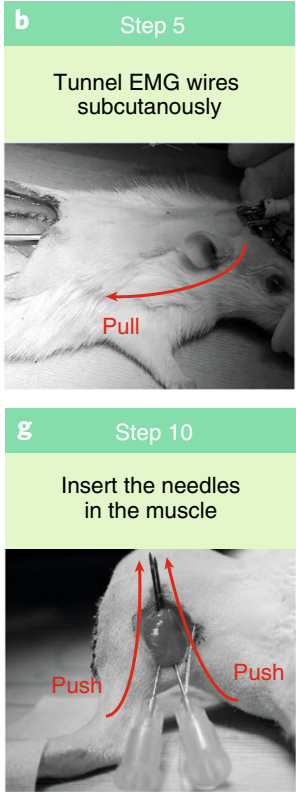
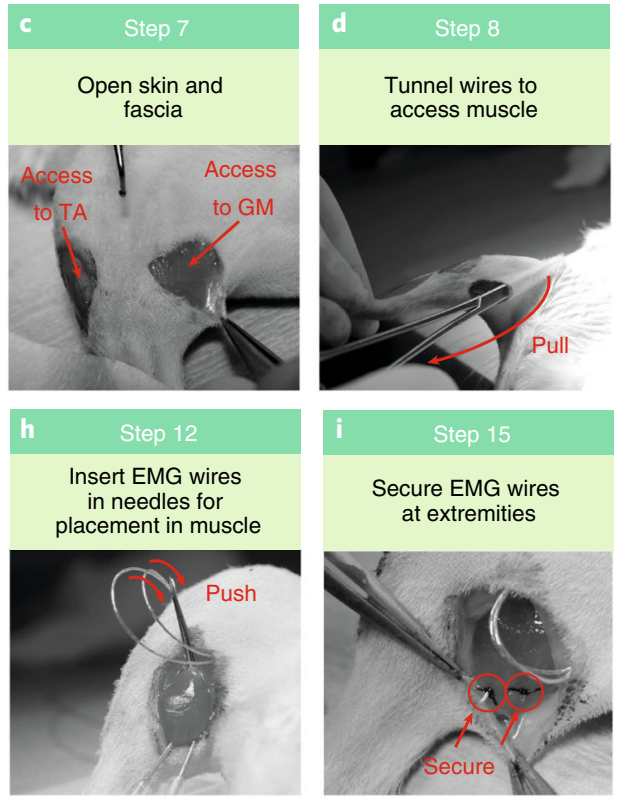
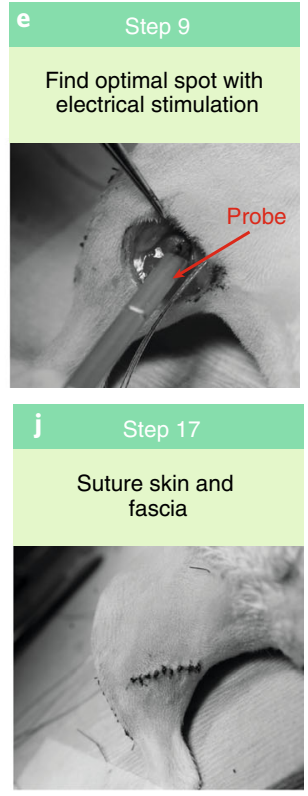

Fig. 3 | Procedure for implantation of chronic EMG leads in rats. a, Prepare two syringe needles ( 24 gauge) by creating a $135^{\circ}$ bend between the tip and the base of the needle. Remove the insulation layer from the silicone-coated stainless-steel wires over a length of $\sim 1 \mathrm{~mm}$, on one side of the wire. The procedure should be carried out using either a microscope (before surgery) or magnification lenses. $\mathbf{b}$, Implant five screws into the skull to form the support for the dental cement plug hosting the head connector. c, Tunnel the EMG wires through the back of the animal. $\mathbf{d}$, Secure the position of the animal and open the skin and the fascia to access the targeted muscles. e, Tunnel the wires coming from the head connector to the implantation point. f, Use an electrical stimulation probe to locate the most contractile spot in the targeted muscle. $\mathbf{g}$, Insert the previously prepared needles into the muscles. The most bent part of the needle should be used as the point of reference for placement of the EMG electrode and should be located around the spot identified in $\mathbf{f}$. $\mathbf{h}$, Insert a pair of wires parallel to the muscle fiber orientation and position the electrode contacts close to the most bent part of the needles. $\mathbf{i}$, Suture the EMG wires to the muscles with simple knots. $\mathbf{j}$, Then close the wound by suturing both the muscle fascia and the skin. All animal experiments depicted in this figure were approved by the local authorities.
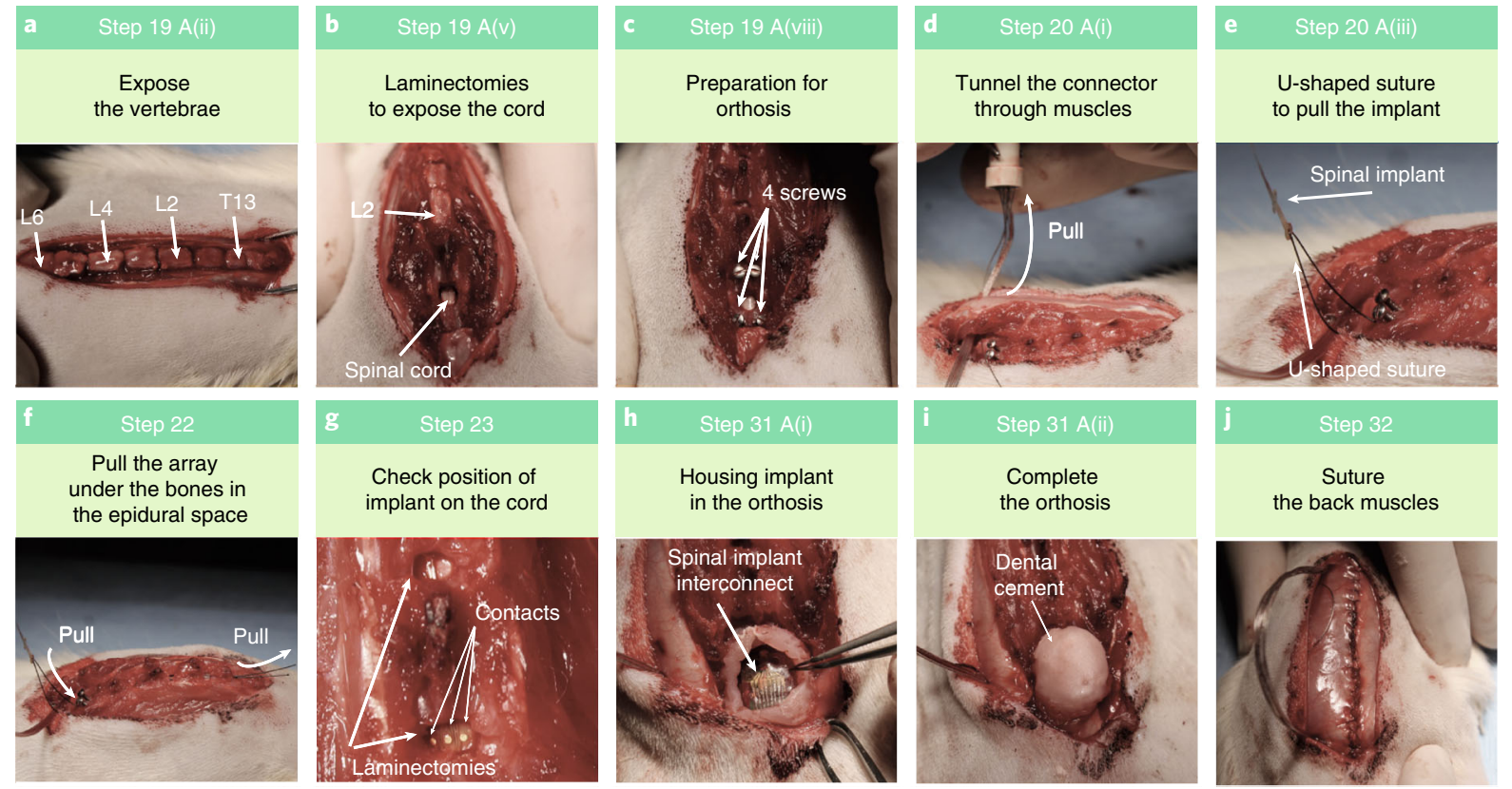

Fig. 4 | Procedure for implantation of the spinal implant in rats. a, Expose the T13 to L6 vertebrae. b, Perform dorsal laminectomies to expose the cord at the insertion point (L3/L4 verterbrae). c, Place four screws on the $L 4$ and $L 5$ vertebrae (two per side) to form a basis for the orthosis hosting the connector. d, Tunnel the head-mounted connector of the implant through an opening in the back muscles. e,f, Use a U-shaped suture (e) to pull the spinal implant beneath the vertebra and above the cord up to the rostral opening at T12-T13 (f). $\mathbf{g}$, Use laminectomies to verify the location of the active sites. h-j. Finally, form the orthosis hosting the connector using dental cement (h,i), then suture the muscles and the skin (j). All animal experiments depicted in this figure were approved by the local authorities. 

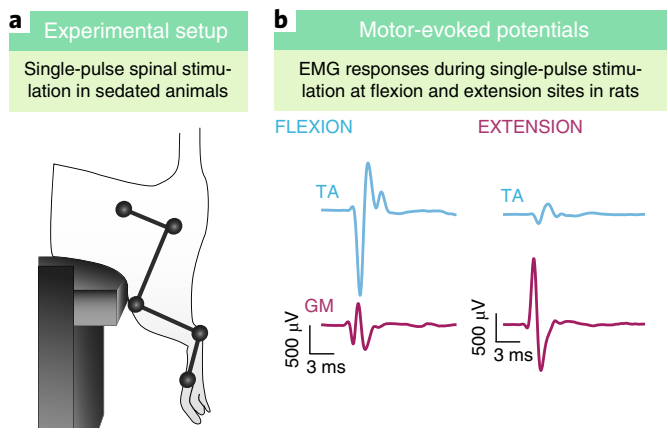

FLEXION EXTENSION
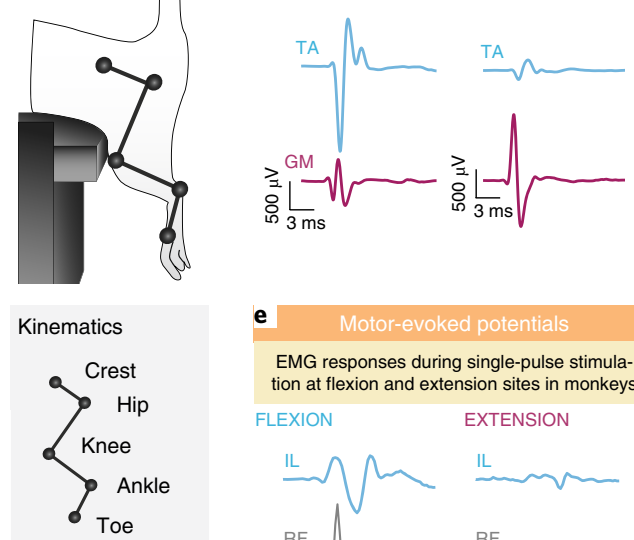

EMG
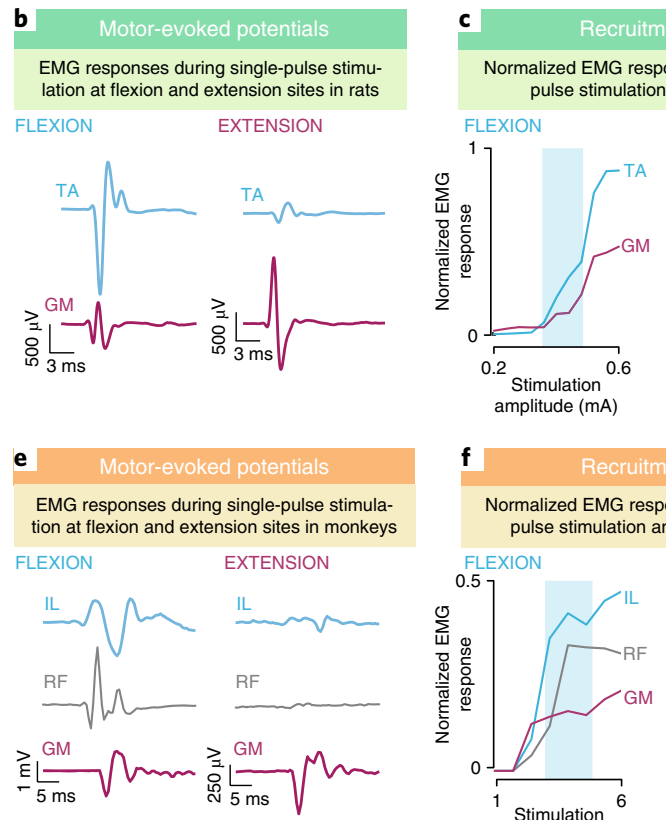

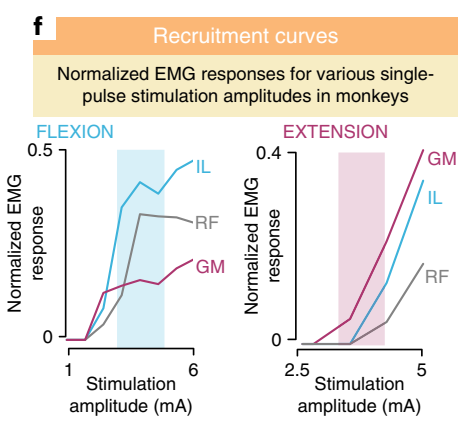

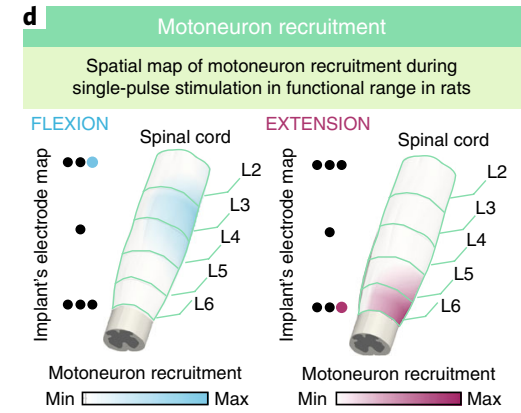

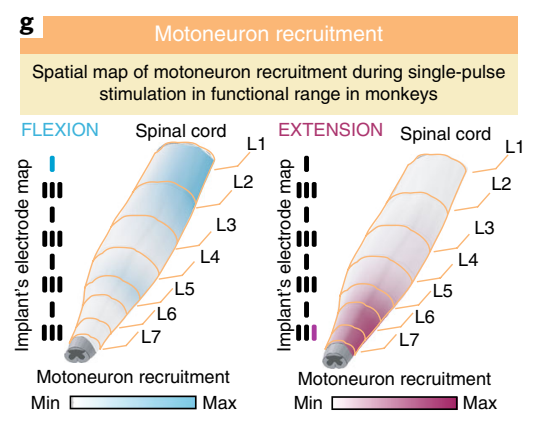

Fig. $\mathbf{5}$ | Spatial and functional specificity of spinal implants. a-g, Summary of the procedure to assess the spatial specificity for selecting 'flexion' and 'extension' electrode configurations in rats (a-d) and Rhesus monkeys (e-g). $\mathbf{a}$, The experimental setup. The animal is sedated and placed in a prone position that allows observation of leg movements. Simultaneous recording of leg joint kinematics can help identify the induced movement (center). Bottom, example of an EMG response evoked by a single EES pulse. b. EMG motor responses evoked by single EES pulses. Stimulating through one of the implant's rostral electrodes (termed 'flexion' electrode) should lead to a higher amplitude of motor response in proximal flexor muscles than in distal extensor muscles. Conversely, stimulating through one of the implant's caudal electrodes (termed 'extension' electrode) should lead to a higher amplitude of motor response in distal extensor muscles than in proximal flexor muscles. Intraoperatively, the longitudinal positioning of the implant should be adjusted to produce results similar to these. c, Muscle recruitment curves measured by delivering single stimulation pulses of increasing amplitude, from motor threshold to saturation of EMG response. The resulting recruitment curves show the specificity obtained for the different stimulation amplitudes, both for the flexion (i.e., rostral) and extension (i.e., caudal) stimulation electrodes. Flexion electrodes should show lower thresholds and larger recruitment of flexor muscles than extensors. Extension electrodes should show the opposite properties. Amplitudes above the motor threshold that preserve the functional specificity of the electrodes define the functional range over which this configuration can be used to promote flexion or extension of the leg. d, Color-coded projection of motoneuron recruitment over the spinal segments. EMG activity is normalized and projected onto the location of the motoneuron pools innervating the leg muscles. Maps show the spots of normalized motoneuron activation for the flexion (white to cyan) and extension (white to magenta) electrodes. The flexion electrodes should activate the more rostral segments, whereas the extension electrodes should activate the more caudal segments within the functional range, as shown in these examples. e-g, Data corresponding to $\mathbf{b}-\mathbf{d}$ for Rhesus monkeys, using the same color-code conventions. c,d adapted with permission from Wenger et al. ${ }^{20}$, Springer Nature Limited; $\mathbf{f}, \mathbf{g}$ adapted with permission from Capogrosso et al. ${ }^{8}$, Springer Nature Limited. All animal experiments depicted in this figure were approved by the local authorities.

the electrode position to target the most rostral and the most caudal spinal segments, which involves targeting the iliopsoas (IL) and GM/soleus muscles, respectively. This longitudinal placement of the spinal implant ensures full coverage of the motoneurons innervating the leg muscles.

\section{Chronic recordings of EMG activity}

Recordings of EMG activity require the acquisition of differential electrical signals with respect to a common ground from a pair of electrodes inserted into the targeted muscle. In both rats and monkeys, silicone-coated stainless-steel wires are appropriate for chronic implantation (Fig. 3). In rats, the wires may be soldered to an off-the-shelf connector that is secured to the skull with screws and dental cement. The same connector may be used to connect the wires from the spinal implant. Any commercial differential amplifier with a high ohmic input (ranging from $100 \mu \mathrm{V}$ to $1 \mathrm{mV}$ ) is suitable to record EMG activity. In monkeys, behavioral experiments involving locomotion require wireless recordings using implanted or head-mounted recording systems. The only commercially available implantable system for recording EMG activity has been discontinued. A few solutions can be implemented to transmit electrical signals to an external receiver using commercially available headstages (e.g., Triangle Biosystems). However, the presence of a percutaneous connector increases the risk of infections. Moreover, the animals may hit the connector against the walls of Plexiglas enclosures, leading to damage of the headstage. EMG recordings are relatively 
stable in both rats and monkeys. Loss of EMG signals can occur due to electrode or system failure. In rats, loss or degradation of EMG signals often occurs when the wires became exposed in proximity to the head-mounted connector, leading to subsequent damage of the wires. In monkeys, the main cause of signal loss is the battery life of fully implantable systems. We used systems with nonrechargeable batteries ${ }^{8}$ that last for up to 1 month of continuous recordings. Depending on usage, the lifetime of recordings can be extended to up 6 months, with several hours of recordings per day.

\section{Temporal specificity and triggering of stimulation protocols \\ Recording of three-dimensional joint kinematics}

To assess gait quality and to extract real-time kinematic feedback, a system to record threedimensional (3D) joint kinematics is needed. The most common motion-capture systems involve video-based recordings of reflective markers (e.g., Vicon Motion Systems) or anatomical landmarks painted on shaved skin (e.g., Simi Reality Motion Systems). The use of reflective markers simplifies their tracking and thus the reconstruction of $3 \mathrm{D}$ positions. However, the markers must be firmly attached to the animal's skin; they are therefore more suited to rats than monkeys. Both types of systems enable offline processing of kinematic data and assessment of gait quality via detailed kinematic analysis. However, currently, only motion-capture systems based on infrared (IR) recordings allow reliable online identification and automatic labeling of joint markers in real time for closed-loop applications, as detailed below.

\section{Spatiotemporal maps of spinal segment activation}

During locomotion of healthy animals, the activation of leg muscles throughout the gait cycle follows a stereotypical sequence of activation patterns, as reflected in highly stereotypical EMG recordings. As the proportion of motoneurons innervating each of the recorded muscles is known for any given spinal segment, this sequence of EMG activity can be translated into a spatiotemporal map of spinal segment activation (Fig. 6$)^{21}$. In both rodents and monkeys, activation of spinal segments across the gait cycle can be divided into a finite number of states, with two (or more) predominant phases corresponding to the movements of flexion and extension. These two states approximately correspond to the onset of the swing and stance phases, respectively, and are therefore referred to as 'foot off and 'foot strike' events. The detection of these specific gait events is essential to triggering the stimulation protocols that promote flexion and extension of the leg. Other states can also be defined to refine the activation of spinal segments. In this protocol, we limit our analysis to these two phases, but the concepts that we describe can also be used to establish more refined protocols ${ }^{49}$.

\section{Detection of gait events}

The 'foot off and 'foot strike' gait events can be extracted from a variety of signal modalities. When the subject is capable of producing some movements, the phases of flexion and extension can be detected from leg kinematics using a motion-capture system or inertial sensors, or from biometric signals such as EMG activity. When the subject is completely paralyzed, these events can be decoded only from brain signals encoding leg movements. Here, we focus on kinematics-based and brainbased detection of gait events. However, regardless of the chosen method, a motion-capture system is necessary for quantifying the quality of the gait and robustness of detections.

\section{Gait event detection: residual kinematics}

Animals with residual motor functions, as observed in individuals with incomplete SCI, remain capable of initiating or attempting leg movements voluntarily. These movements can be detected from kinematic recordings, using an online motion-capture system (e.g., Vicon Motion Systems). Even in rats with complete SCI, automated stepping-like leg movements can beelicited when the legs are moved backward in response to treadmill belt motion, or when pinching the tail. To detect leg movements, the subject is placed within a recording space defined by a set of IR cameras that cover the desired 3D volume. The cameras track IR-reflective markers that are attached to the subject's skin overlying anatomical landmarks (e.g., crest, hip, knee, ankle and metatarsal joints, Fig. 1).

The $3 \mathrm{D}$ position of each marker is reconstructed in real time, using commercially available software and routines that allow linking of each marker to the joint of a body model-a process called 'labeling' in commonly used motion-tracking software (e.g., Nexus Software, Vicon). 

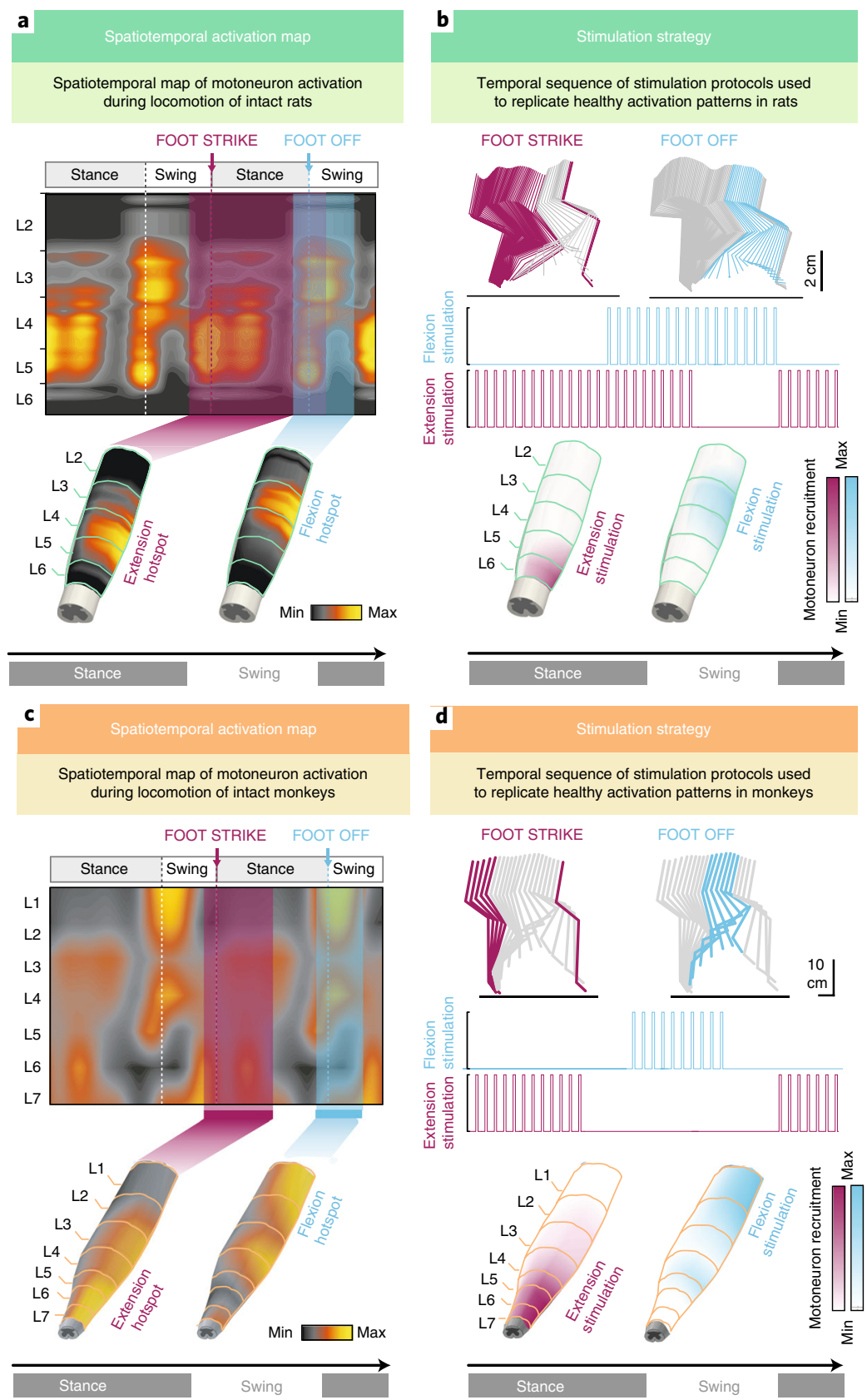

Fig. 6 | Spatiotemporal neuromodulation of the lumbar spinal cord. a-d, For both rats $(\mathbf{a}, \mathbf{b})$ and Rhesus monkeys (c, d), the natural sequence of activation of motoneuron pools during locomotion is used to derive stimulation protocols that reproduce this sequence after injury. Stimulation protocols to modulate the extension and flexion movements of the legs are timed to kinematic events. a (top), Spatiotemporal map of motoneuron activation during locomotion in rats. Recordings of EMG activity from leg muscles during locomotion of intact animals are projected onto the spatial location of the corresponding motoneurons in the spinal cord. This allows the identification of natural spatiotemporal patterns of motoneuron activation in the lumbar spinal cord. This map is composed of bursts of activation that span the rostral and caudal segments of the lumbosacral spinal cord (bottom). b. Stimulation protocols are applied, mimicking the natural sequence of motoneuron activation, based on the spatial maps obtained with single EES pulses (Fig. 4). From these maps, a temporal sequence of stimulation bursts is calculated. These bursts are synchronized to appropriate kinematic events along the gait cycle. Top, stick diagrams representing leg segments during walking are color coded according to the active stimulation electrode; magenta indicates extension and cyan indicates flexion. Below is the corresponding stimulation protocol unfolded over time and relative to the stance and swing phases. Bottom, spatial recruitment of motoneurons for the extension (magenta) and flexion (cyan) electrodes. These patterns mimic the spatial activation of motoneurons during the stance and swing phases of the gait cycle. $\mathbf{c}, \mathbf{d}$, Corresponding data for Rhesus monkeys, using the same color-coding conventions. a,b adapted with permission from Wenger et al. ${ }^{20}$, Springer Nature Limited; c,d adapted with permission from Capogrosso et al. ${ }^{8}$, Springer Nature Limited. All animal experiments depicted in this figure were approved by the local authorities. 

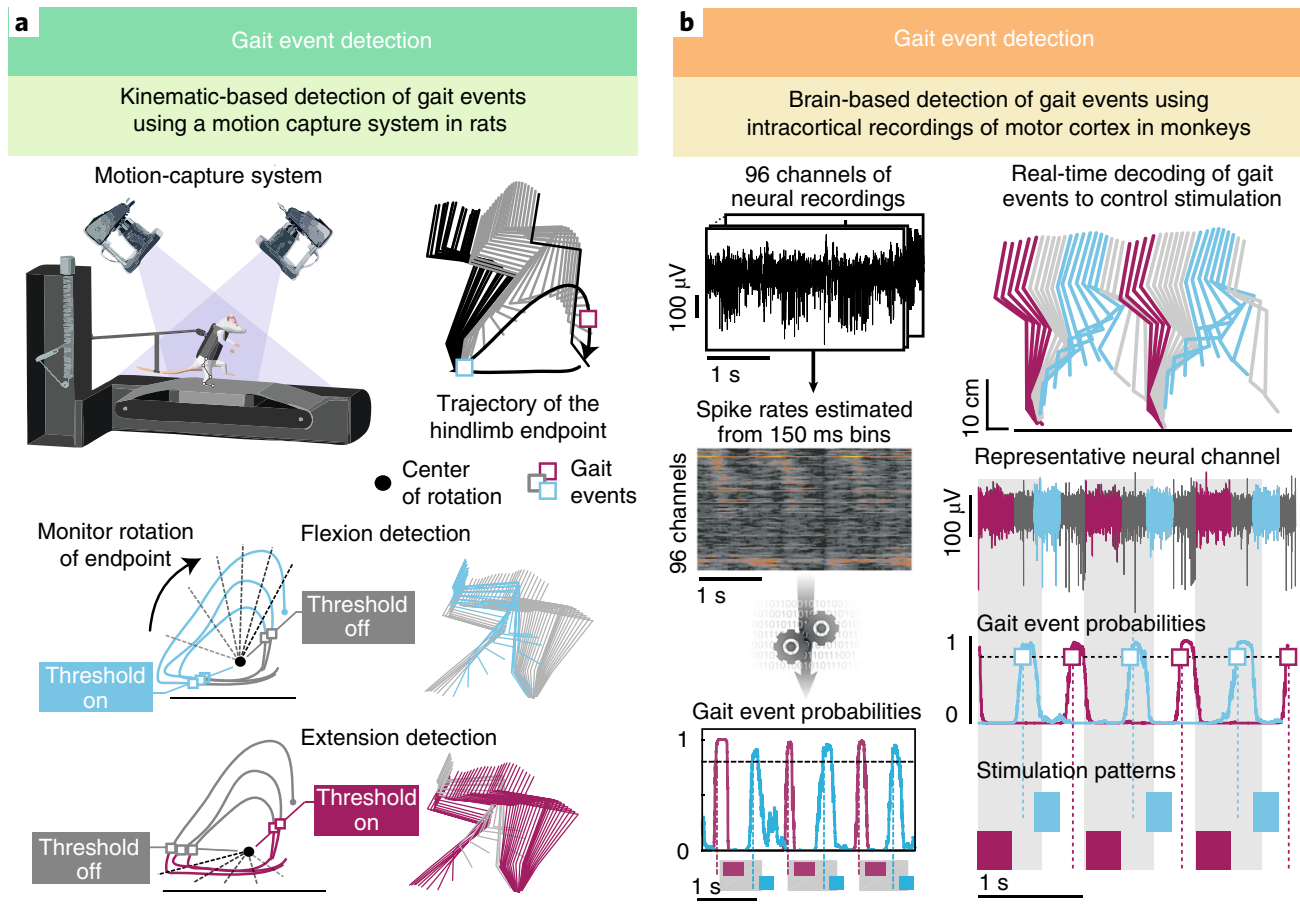

Fig. 7 | Real-time detection of gait events. The two main gait events necessary to trigger the spatiotemporal stimulation protocols are the 'Foot off' and 'Foot strike' events. a, In rats, these events are detected by defining the threshold angles on the 2D trajectory of the ankle marker during gait. Top, the trajectory is computed by projecting the $3 \mathrm{D}$ position of the ankle marker along the sagittal plane. Bottom, flexion and extension stimulation bursts are triggered at 'Threshold on' events and stopped at 'Threshold off' events. 'Threshold on' timings correspond to either the 'Foot off' and 'Foot strike' events. Event detections are shown in the figure by the cyan and magenta boxes for 'Foot off' and 'Foot strike', respectively. The leg joint position corresponding to these phases is shown on the bottom panel; magenta and cyan stick segments represent time intervals in which extension and flexion stimulation is on, respectively. b, In monkeys, the gait events are detected (or predicted) from the decoding of intracortical activity. Stimulation bursts are then triggered upon detection of these gait events. On the left, the computational flow is shown. Multi-unit spike events are extracted, and firing rates are estimated for each of the recorded channels. Firing rates are then used to compute the probability of occurrence of 'Foot off' and 'Foot strike' events. Event detection occurs upon crossing a 0.80 probability threshold. Right, stick diagrams of two consecutive steps. Bottom, an example of a raw neural recording, the corresponding probability of 'Foot off' and 'Foot strike' events and stimulation patterns triggered upon detection. Color coding is as in $\mathbf{a}$. a adapted with permission from Wenger et al. ${ }^{20}$, Springer Nature Limited; $\mathbf{b}$ adapted from Capogrosso et al. $^{8}$, Springer Nature Limited. All animal experiments depicted in this figure were approved by the local authorities.

Various kinematic parameters may be used to detect gait events. From our experience, the most robust method of extracting the 'foot off and 'foot strike' events is based on monitoring the position of the ankle marker over time. Specifically, we project the 3D trajectory of this marker onto the $z-x$ plane (sagittal), in which $x$ is the plane corresponding to the direction of locomotion and $z$ is the vertical axis (Fig. 1). When the trajectory of the ankle marker is represented with respect to a fixed position on the body-for example, the crest or the hip position-it describes a stereotypical ellipsoidal trajectory that captures many of the key spatiotemporal features underlying gait movements. To extract these features, we calculate the instantaneous angle of rotation of the foot with respect to the center of gravity of the entire trajectory ${ }^{20}$ (Fig. 7). This angle provides a measure of the current phase throughout the gait cycle and naturally accounts for gait periodicity. To additionally account for changes in kinematic features through time due to various factors such as natural muscle fatigue, loss of motivation and intrinsic variability of the gait cycle, it is recommended that estimates of the center of gravity be continuously updated. After each step, each new observation can be used to iteratively adapt such estimates, using adaptive filters.

We set the reference frame to ensure that the trajectory of the ankle marker evolves clockwise, and that the 'foot strike' and 'foot off' events occur at $\sim 0$ and $180^{\circ}$, respectively (Fig. 7). In turn, the events are defined when the value of the angle crosses the exact thresholds defined by the experimenter. Note 
that it is important to fine-tune these thresholds depending on the subject and the specific walking condition (e.g., body-weight support), as described in more detail later.

Gait event detection: neural signals

Gait events can also be detected from brain signals that encode movement. A range of different electrophysiological techniques can be used to decode gait parameters, including non-invasive EEG recordings ${ }^{50-53}$, electrocorticographic (ECoG) signals recorded using electrodes positioned on the cortical surface $e^{25,54,55}$, and local field potentials and action potentials of neuronal ensembles recorded using electrodes implanted into the cortex ${ }^{8,56,57}$. Generally, the decoding accuracy and robustness increase with the increasing spatial resolution ${ }^{58}$. On the basis of our experience, high accuracy and robustness can be achieved using the activity of neuronal ensembles recorded using microelectrode arrays implanted into the leg area of the primary motor cortex $^{8}$. Typically, these implants cover a $4 \mathrm{~mm} \times 4$-mm area of the cortex, with 96 penetrating electrodes. Although experiments with monkeys using tethered systems are possible ${ }^{56}$, the range of movements and behavioral tasks increase when the subjects are free to move naturally, leading to more behaviorally and clinically relevant data. To support such experimental paradigms, wireless transmission of neural and other signals is necessary ${ }^{59-62}$. Such microelectrode arrays chronically implanted into the cerebral cortex of nonhuman primates have provided reliable signals for durations of up to 1.5 years ${ }^{63,64}$. Numerous clinical case studies reported useful neural recordings for even longer periods ${ }^{65}$, albeit the quality and content of the signals declined over time ${ }^{66}$.

Here, we focus on describing the use of intracortically recorded activity of neuronal ensembles, using microelectrode arrays to detect gait events. For each electrode, the acquired recordings are band-pass-filtered between $500 \mathrm{~Hz}$ and $7.5 \mathrm{kHz}$ to isolate the multiunit spiking activity. A 10 -s period of recordings is used to estimate the standard deviation of the filtered signal for each electrode. The system marks each time the filtered signal crosses a threshold, set to a value typically between -3 and -5 standard deviations. These threshold crossings are denoted as 'multiunit spikes'. Finally, the number of these spikes within the past $100-200 \mathrm{~ms}$ is used to estimate the current spike rate. The kinematic or muscle activity synchronized with the neural signals can be used to identify time points within the spike rate signals that correspond to discrete gait events, such as 'right foot off and 'right foot strike' events. These labeled neuronal signals can be used to calibrate a decoder that detects gait events from current and past spike-rate signals. For example, a regularized linear or quadratic discriminant analysis classifier can be calibrated using the labeled spiking rates and then adapted to detect discrete events from continuous neural signals. In this condition, the decoder outputs the probability of each of the gait events, given the current and past spike rates. When one of these probabilities crosses a level ranging from 0.5 to 0.99 , the corresponding gait event will be detected and can be used to trigger stimulation protocols (Figs. 1 and 8).

For configuring spatiotemporal stimulation protocols, the decoder should be trained off-line to detect the events 'foot off and 'foot strike'. Real-time estimation of the probabilities of these events will be used for online detection and subsequent triggering of the flexion or extension stimulation pattern (Fig. 7). The detailed implementation of such decoders has been described previously in more detail ${ }^{8,67}$. A similar procedure, consisting of a processing part that derives decoder inputs from the recorded brain signals, and the decoding part, can be used with other signal modalities.

One important aspect to consider when using neural signal decoding in combination with EES is the contamination of cortical signals with sensory-evoked potentials elicited by the recruitment of dorsal column and dorsal root fibers after each pulse of stimulation ${ }^{68}$. Because of this contamination, gait decoders should be trained in the presence of stimulation, using a two-stage training of the decoder: first without stimulation and then with stimulation triggered by the decoder obtained from the first stage ${ }^{8}$.

Duration of stimulation protocols

Although the detection of 'foot off and 'foot strike' events is sufficient to define the onset of the flexion and extension stimulation protocols, the duration of the corresponding stimulation requires optimization. Among all the procedures described here, the identification of the appropriate onsets and durations of each stimulation burst is the most sensitive part of the protocol. These parameters are derived from several experimental sessions during which the effects of different parameter 


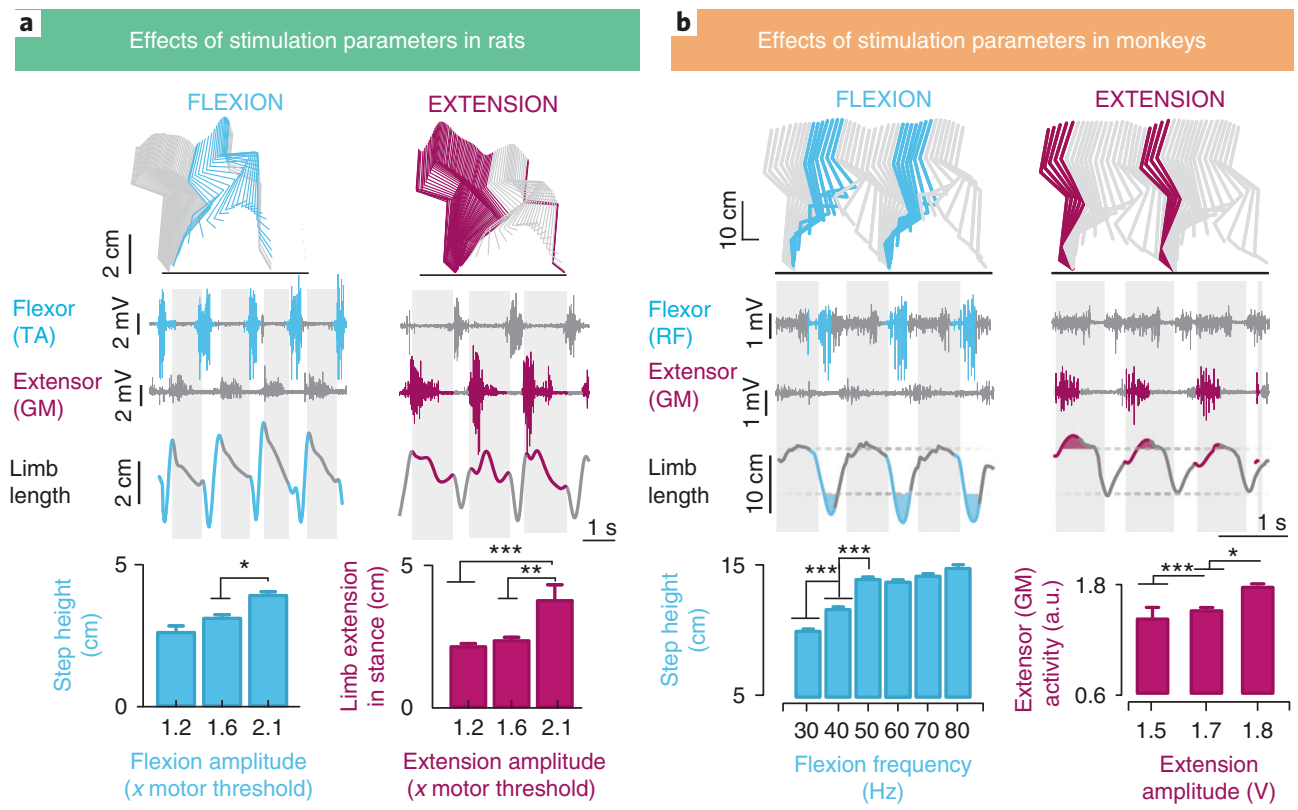

Fig. 8 | Amplitude and frequency modulation of kinematic and EMG activity during walking enabled by spatiotemporal neuromodulation. a,b, For both rats (a) and monkeys (b), changing stimulation parameters such as frequency or amplitude of either the flexion or extension stimulation mediates substantial modulation of both leg kinematics and EMG activity. This modulation guides the optimization of stimulation parameters, with the aim to promote leg kinematics resembling the patterns observed in intact animals or to modulate leg kinematics to produce adaptation of leg movements, for example, to climb a staircase. All statistics represent the effect of stimulation amplitude or frequency on kinematics or EMG for a representative animal, corresponding to the raw data shown above. Bar graphs and error bars indicate, respectively, the mean and s.e.m. of the plotted variable across all available steps. Left-hand bar chart in $\mathbf{a}: n=19,23$ and 17 steps for amplitudes of 1.2, 1.6 and 2.1, respectively. Right-hand bar chart in a: $n=21,16$ and 12 steps for amplitudes of 1.2, 1.8 and 2.1, respectively. Lefthand bar chart in $\mathbf{b}: n=20$ steps for each frequency. Right-hand bar chart in $\mathbf{b}: n=13,27$ and 13 steps for amplitudes of 1.5, 1.7 and 1.8, respectively. All pairwise comparisons were performed using Wilcoxon rank-sum tests. ${ }^{\star} P<0.05,{ }^{\star \star} P<0.01,{ }^{\star \star \star} P<0.001$. a adapted with permission from Wenger et al. ${ }^{20}$, Springer Nature Limited; $\mathbf{b}$ adapted from Capogrosso et al. ${ }^{8}$, Springer Nature Limited. All animal experiments depicted in this figure were approved by the local authorities.

combinations on leg kinematics are studied systematically ${ }^{20}$. To identify the optimal parameters, a broad range of variables quantifying gait patterns are calculated using the 3D kinematic data acquired during the experiments. The entire set of variables defines a multidimensional space in which the Euclidean distance from a healthy reference gait quantifies the performance of each combination of stimulation parameters ${ }^{8}$. The minimal distance from the healthy reference identifies the optimal parameter combination.

The duration of stimulation bursts can either be pre-programmed or terminated by a dedicated gait event. In the latter case, the duration of each stimulation burst is automatically adjusted to the duration of the current gait cycle. The procedure used to perform this optimization is discussed in more detail in Part III of the Procedure.

\section{Limitations}

Spatiotemporal neuromodulation therapies applied to the lumbar spinal cord have been remarkably successful in ameliorating gait after SCI in animal models ranging from rodents to nonhuman primates. However, the degree of facilitation was contingent on the severity of the injury. After the most severe types of SCI, EES alone mediates alternating movements of the legs, but the animals demonstrate limited weight-bearing capacities and poor foot placement. To remedy this limitation, it is essential to deliver an additional serotonergic replacement therapy that tunes the excitability of spinal circuits to a level that augments their responsiveness to electrical stimulation and other sources of inputs ${ }^{13,24,69}$.

The reliability of gait event detections is an additional limitation of the current protocol. The accuracy and robustness of the decoded events are directly contingent on the quality of locomotor 
movements. Consequently, gait events are difficult to decode from leg kinematics in severely paralyzed animals. Similar limitations apply to the decoding of gait events from brain signals. When the extent of residual leg movements is limited, optimal training of the decoder is hindered by the absence of overt movements correlated to motor intents. This results in decreased performance of the decoder.

\section{Comparison with other technologies for the restoration of motor control Intraspinal microstimulation of the spinal cord}

Intraspinal microstimulation (ISMS) consists of the application of electrical currents directly inside the gray matter of the spinal cord by means of arrays of microelectrodes. This technique has been applied to produce coordinated movements of arm and leg muscles, using a limited number of electrode contacts in several animal models ${ }^{49,70-73}$. Although the electrical field induced by ISMS is more focal than the field elicited by EES, the resulting activation of neural structures is not necessarily more specific. Due to the lower threshold of axons as compared with cell bodies ${ }^{74}$, ISMS recruits fibers of passage from sensory afferents at lower or similar thresholds than motoneurons ${ }^{75,76}$. However, primary sensory afferents located within a given spinal segment originate from multiple dorsal roots that converge on the targeted motoneurons ${ }^{77-79}$ located over multiple segments ${ }^{73}$. This anatomical organization results in limited muscular specificity and unclear spatial relationships between electrode locations and recruited muscles ${ }^{70}$. Conversely, EES activates dorsal root afferents before they enter to the spinal cord ${ }^{10}$. Primary afferents associated with a given muscle are mainly grouped within one or two roots. Therefore, the recruitment of a dorsal root will activate only the muscles that receive monosynaptic or polysynaptic inputs from the fibers embedded in this root. Consequently, there is a strict anatomical relationship between electrode location, recruited root and recruited muscles.

Functional electrical stimulation of muscles

Functional electrical stimulation of muscles (FES) is a clinically approved method for engaging paralyzed muscles ${ }^{80}$. FES protocols have reanimated paralyzed arm muscles in nonhuman primates $^{81}$ and people with tetraplegia, using both superficial ${ }^{82}$ and implanted electrodes ${ }^{83}$. Similar results have been obtained with leg musles ${ }^{80}$. In contrast to EES and ISMS, the electrical currents delivered by FES directly activate the efferent fibers or muscle fibers. This paradigm thus bypasses the sensorimotor circuits in the spinal cord. This feature is an appealing approach to restoring functions after paralysis, as movements can be produced without any contribution from sensory feedback or residual descending control. However, such stimulation leads to fast muscle fatigue due to the inverse recruitment order of efferent fibers. As fibers with large diameter are recruited before those with smaller diameters, FES quickly leads to fatigue during sustained stimulation ${ }^{84}$. The production of coordinated movements with FES requires artificial coordination of multiple muscles in time, which poses a substantial challenge for control engineering $^{85}$. Instead, EES recruits motoneurons trans-synaptically ${ }^{10,11}$, thus recruiting them in a natural order. EES can enable locomotion over extensive periods of time in animal models of paralysis ${ }^{23}$.

\section{Overview of the procedures}

For clarity, we have divided all procedures necessary for the configuration of spatiotemporal neuromodulation into three main parts.

Part I-implantation of EMG electrodes into leg muscles and spinal implants over the lumbosacral spinal cord in rats and nonhuman primates (Steps 1-35);

Part II-establishment of spatial specificity (comprising post-surgical selection of optimal electrode configurations; Steps 36-49);

Part III-establishment of time specificity (comprising determination of stimulation patterns; Steps 50-57).

The use of intracortical microelectrode arrays to decode gait events from brain signals is optional. The corresponding implantation protocols have been described in detail elsewhere ${ }^{64,86}$ and are therefore not included in the current article. Experimenters who wish to use brain signals for gait event decoding should follow these specific protocols before starting Part III of the following Procedure. 


\section{Animals}

- Rats: We have used female Lewis rats (Janvier Labs, strain code LEW-ORIj), initial weight 180-220 g. ! CAUTION All experiments on rodents must conform to national and institutional regulations. All the procedures described here for rats are in accordance with Swiss federal legislation and were established under the guidelines established at EPFL (authorization no. VD2771). Local Swiss Veterinary Offices also approved all the procedures. $\triangle$ CRITICAL Female Lewis rats are particularly suited to the protocols described, due to their behavioral stability and ease of handling. Females are preferred, as the bladder is easier to void than in males.

- Primates: We have used Rhesus macaques, males, 4-9 years old, with average weight of $6.5 \pm 0.5 \mathrm{~kg}$. ! CAUTION All use of primates must conform to national and institutional regulations. The experimental protocol on primates we describe here was approved by the Institutional Animal Care and Use Committee of Bordeaux (CE50, France) under license number 50120102-A and by the cantonal (Fribourg) and federal (Swiss) veterinary authorities (authorization no. 2016_09_FR). The experiments for which we show results to illustrate this protocol were performed in accordance with the European Union directive of 22 September 2010 (2010/63/EU) on the protection of animals used for scientific purposes in an AAALAC- accredited facility (Chinese Academy of Science, Beijing, China). $\triangle$ CRITICAL As an alternative to large male Rhesus macaques, smaller Macaca fascicularis females can also be used (3.5-4.5 kg). M. fascicularis females are much more docile and collaborative than large males; however, they are behaviorally more unstable. The use of females is recommended in facilities with group housing.

\section{Reagents}

- Ethylene oxide (custom order, requires accredited facilities, not possible to use in academic laboratory)

- 70\% ethanol (Gilbert Laboratories, cat. no. B01CZ5XP2W)

- Medetomidine (Domitor, Henri Schein, cat. no. 8402)

- Carprofen (Rimadyl, Henri Schein, cat. no. ZO10002249)

- Isoflurane (Attane Isoflurane, Henri Schein, cat. no. 2222)

- Pure oxygen (Carbagas Switzerland, cat. no. A1CTPX7)

- Ketamine ((Ketasol-100, Graeub; Swissmedic no. 50375)

- Benzodiazepine, midazolam (Dormicum; Roche Pharma; Swissmedic no. 44448)

- Methadone (Methadon Streuli, Streuli Pharma; Swissmedic no. 34383)

- Propofol (Henri Schein, cat. no. 4990)

- Fentanyl (Fentanyl Curamed, Mepha Pharma; Swissmedic no. 53484)

- Ophthalmic ointment (Lacryvisc, Alcon Switzerland; Swissmedic no. 54944)

- Dental cement (Alphacryl, Rapid Repair, Cold-Cure Denture Base, National Dental Supplies)

- Ringer's solution (Henri Schein, cat. no. 3446)

- Buprenorphine (Temgesic, Indivior, Baar; Swissmedic no 41931)

- Amoxicillin (Noroclav, Ufamed; Swissmedic no. 57'024)

\section{Equipment}

\section{Surgical tools for rats}

- Micro-mosquito hemostat, straight (Fine Science Tools, cat. no. 13010-12)

- Halsted mosquito hemostat, straight (Fine Science Tools, cat. no. 13008-12)

- Olsen-Hegar needle holder, tungsten carbide (Fine Science Tools, cat. no. 12502-12)

- Adson forceps, with teeth (Fine Science Tools, cat. no. 11027-12)

- Student Adson forceps, serrated (Fine Science Tools, cat. no. 91106-12)

- Dumont no. 2 laminectomy forceps (two pair; Fine Science Tools, cat. no. 11223-20)

- Extra-fine Bonn scissors, straight (Fine Science Tools, cat. no. 14084-08)

- Friedman Pearson rongeur, 0.5-mm cup (Fine Science Tools, cat. no. 16221-14)

- Rongeur, 1-mm cup (Allgaier Instrumente, cat. no. 31-323-150)

- Student scalpel handle (Fine Science Tools, cat. no. 91003-12)

- Hemostatic clamp (Henri Schein, cat. no. 120718, 120720 or 122016)

- Needles for EMG placement, 23 gauge, 0.6-mm diameter, 30-mm length (Henri Schein, cat. no. 602104)

- Dumont no. 5/45 forceps (two pair; Dumoxel; Fine Science Tools, cat. no. 11251-35)

- Student Dumont forceps (Fine Science Tools, cat. no. 91150-20)

- Dental plugger (Henri Schein, cat. no. 9001672) 
- Sutures (Ethilon 4.0; Henri Schein, cat. no. 168660)

- Liquid plastic welding (Histoacryl, Henri Schein, cat. no. 1050052)

- Gauze (Henri Schein, cat. no. 604767) or cotton pellets (Henri Schein, cat. no. 9001879)

- Stainless-steel self-tapping screws (diameter $=0.7 \mathrm{~mm}$; Antrin Miniature Specialties, cat. no. 000FN68PI)

Surgical tools for monkeys

- Pean forceps, curved, $14 \mathrm{~cm}$ (Kruuse Rochester, cat. no. FORPS KC14)

- Pean forceps, straight, $14 \mathrm{~cm}$ (Kruuse Rochester, cat. no. FORPS KS14)

- Maxima Mayo-Hegar needle holder, tungsten carbide, $16 \mathrm{~cm}$ (Henry Schein, cat. no. 146516)

- Scalpel blade, no. 10 (Henry Schein, cat. no. SCAL HS10)

- Scalpel blade, no. 11 (Henry Schein, cat. no. SCAL HS11)

- Maxima Spencer suture scissors, straight, $11 \mathrm{~cm}$ (Henry Schein, cat. no. SCSS M20)

- Maxima iris scissors, straight, $9 \mathrm{~cm}$ (Henry Schein, cat. no. SCIS M10)

- Needles for EMG placement, 23 gauge, 0.6-mm diameter, 60-mm length (Henri Schein, cat. no. 602122)

- Dumont no. 5/45 forceps (two pair, Dumoxel; Fine Science Tools, cat. no. 11251-35)

- Student Dumont forceps (Fine Science Tools, cat. no. 91150-20)

- Dumont no. 2 laminectomy forceps (two pair; Fine Science Tools, cat. no. 11223-20)

- Extra-fine Bonn scissors, straight (Fine Science Tools, cat. no. 14084-08)

- Maxima Adson dressing forceps, $12 \mathrm{~cm}$ (Henry Schein, cat. no. FOAP M10)

- Adson tissue forceps, $1 \times 2$ teeth, $12 \mathrm{~cm}$ (Henry Schein, cat. no. FOA12 HS11)

- Scalpel handle, no. 3 (Henry Schein, cat. no. SCAL H HS3)

- Surgical spreader (retractor), $130 \mathrm{~mm}$, blunt, $3 \times 4$ teeth (Henri Schein, cat. no. 310487)

- Maxima Doyen forceps, straight, $22 \mathrm{~cm}$ (Henry Schein, cat. no. FODIS M20)

- Explorer Se, no. 8, octagonal (Henry Schein, cat. no. DENT H1660)

- Surgical helmet, 3.0× magnification, $460 \mathrm{~mm}$ (VisionMag, cat. no. VAW4020304)

- Rongeur, $180 \mathrm{~mm}, 130^{\circ}, 2 \mathrm{~mm}$, thin (Kerrison, cat. no. FK907R)

- Spinal insertor (Medtronic, custom order for research purposes only)

- Rongeur, Luer, $150 \mathrm{~mm}$, bent, fine jaws (Henri Schein, cat. no. 210402)

- Micro needle holder, bent, block, $14 \mathrm{~cm}$ (Castroviejo, cat. no. 149114)

- Surgical drill (Aesculap, model no. Elan 4)

- Pneumatic electrode inserter system (Blackrock Microsystems, model no. 7281)

- Screwdriver (DePuy Synthes, cat. no. 03.503.201)

- Sutures (Ethilon 4.0; Henri Schein, cat. no. 168660)

- Liquid plastic welding (Histoacryl, Henri Schein, cat. no. 1050052)

- Stereotaxic frame (Kopf Instruments, cat. no. 1404)

- Soft pads (Henri Schein, cat. no. 9008132)

- Gauze (Henri Schein, cat. no. 604767) or cotton pellets (Henri Schein, cat. no. 9001879)

- Titanium self-drilling screws (6 mm long with 1.5-mm diameter; Synthes, cat. no. 04.503.226.04C)

- Titanium orthosis (custom made)

- Stimulation probe (Neuroline Bipolar probe, Ambu, cat. no. 73602-190/10)

Wired recordings of electromyographic activity in rats

- BioAmp processor (Tucker-Davis Technologies, cat. no. RZ2)

- NeuroDigitizer, minimum 32 channels (Tucker-Davis Technologies)

- Silicone-coated stainless-steel wires (Cooner Wire, cat. no. AS631)

- 16-pin round circular connectors (Omnetics, cat. no. A79112-001)

Wired epidural electrical stimulation in rats

- 16-channel stimulator (Tucker-Davis Technologies, cat. no. IZ2H)

- Isolated pulse stimulator (AM-Systems, model no. 2100), for muscle probe and high-impedance spinal implants

-16-pin round circular connectors (Omnetics, cat. no. A79112-001)

\section{Kinematic recordings in rats}

- Motion-capture system consisting of 12 or more Vero IR cameras, an MX Giganet acquisition module and the Nexus software suite (Vicon, custom order)

- Treadmill equipped with a body-weight-support system (e.g., Robomedica, model no. RR-ATM and RR-BWS) 
Wired recordings of electromyographic activity in monkeys

- BioAmp processor (Tucker-Davis Technologies, cat. no. RZ2)

- NeuroDigitizer, minimum 32 channels (Tucker-Davis Technologies, cat. no. PZ5)

- Silicone-coated stainless-steel wires (Cooner Wire, cat. no. AS631)

- Headstage (Triangle Biosystems, model no. W16)

Wired epidural electrical stimulation in monkeys

- 16-channel stimulator (Tucker-Davis Technologies, cat. no. IZ2MH)

- Isolated pulse stimulator (AM-Systems, model no. 2100), for muscle probe and high-impedance spinal implants

- Insulated crocodile clips, maximum 4-mm width (Ponoma Electronics, cat. no. 5914), for clamping electrode connector during intra-operative mapping

-18-pin nano-strip linear connectors with 2 guide posts (Omnetics, cat. no. A79038-001)

Wireless epidural electrical stimulation in monkeys

- Implantable pulse stimulator (Activa RC; Medtronic, custom order for reserach purpose only)

- Neural Research Programmer application (Medtronic, custom order for research purposes only)

Kinematic recordings in monkeys

- Motion-capture system consisting of six video cameras and associated software suite (SIMI, model no. Simi-Motion)

- N-Mill treadmill (ForceLink, model no. N-Mill)

- Custom-built treadmill with Plexiglas enclosure (custom order)

Wireless recordings of brain activity in monkeys

- 128-channel Cerebus System (Blackrock Microsystems)

- CerePlex W, wireless system (Blackrock Microsystems, model no. CerePlex W)

-96-microelectrode arrays (Blackrock Microsystems)

Wireless recordings of electromyographic activity in monkeys

- Implantable differential RF amplifier (Konigsberg)

- W-series receiver, 16 channels (Triangle Biosystems, model no. W16)

-W-series transmitter, 16 channels (Triangle Biosystems, model no. W16) ! CAUTION The only commercially available implantable system for recording EMG activity has been discontinued. A few solutions can be implemented to transmit electrical signals to an external receiver using commercially available headstages (e.g., those from Triangle Biosystems). However, custom procedures would need to be developed.

\section{Procedure}

\section{Part I: Preparation of animals for surgery Timing $\sim 30$ min}

1 House and handle the animals according to institutional and national guidelines for animal care and use until they reach a size appropriate for implantation. Appropriate sizes are $200-300 \mathrm{~g}$ for rats and 3.5-6.5 kg for Macaque monkeys.

! CAUTION All experiments using animals should be carried out according to institutional and national guidelines.

2 Ensure that all surgical tools are clean and sterile. Disinfect the area with $70 \%$ (vol/vol) ethanol and sterilize all tools by autoclaving. Sterilization of fine surgical tools, spinal implant and EMG electrodes can be performed using ethylene oxide or by plasma sterilization.

$\triangle$ CRITICAL STEP Perform all surgeries under aseptic conditions. It is preferable to use human standards for sterilization and aseptic surgery when implanting electrodes and devices for extended periods of time, especially in monkeys.

3 Sedate rats as described in option A and monkeys as described in option B.

(A) Sedation of rats

(i) Sedate with medetomidine (Dorbene, $0.5 \mathrm{mg} / 100$ g, s.c.) and inject carprofen (Rimadyl, 5 $\mathrm{mg} / \mathrm{kg}$, s.c.) to provide intraoperative analgesia.

(ii) After $5 \mathrm{~min}$, induce anesthesia by placing the rat in a box prefilled with isoflurane (4-5\% in pure oxygen). 
(iii) Place the animals on a heating pad to maintain the body temperature at $37{ }^{\circ} \mathrm{C}$ for rats. Using a nose cone, maintain anesthesia using 1-2\% isoflurane mixed with pure oxygen (oxygen flow rate: $0.8 \mathrm{l} / \mathrm{min}$ ).

$\triangle$ CRITICAL STEP Check for the absence of toe-pinch reflex before proceeding to the next step.

(B) Sedation of monkeys

(i) Sedate with ketamine $(10 \mathrm{mg} / \mathrm{kg})$, benzodiazepine $(0.1 \mathrm{mg} / \mathrm{kg})$ and methasone $(0.2 \mathrm{mg} / \mathrm{kg})$, administered via a single i.m. injection.

(ii) 15-20 min after induction of the anesthesia, place the animals on a heating pad to maintain the body temperature at $35-37^{\circ} \mathrm{C}$. Intubate the animal and maintain anesthesia using $1-3 \%$ isoflurane in pure oxygen at $0.5-1.1$ liters/min or continuous intravenous perfusion of propofol $(0.1-0.4 \mathrm{mg} / \mathrm{kg} / \mathrm{min})$.

! CAUTION In monkeys, the best practice is to use an anesthesia machine to ventilate the animal and for continuous monitoring of oxygen saturation, heart rate, body temperature and respiratory rate.

$\triangle$ CRITICAL STEP Intravenous injection of fentanyl should also be administered during the most painful surgical procedures $(0.1-0.7 \mu \mathrm{g} / \mathrm{kg} / \mathrm{min})$, as indicated later in the procedure. $\triangle$ CRITICAL STEP Continuously monitor the breathing of the animal throughout the surgery, and regulate the flux and concentration of the isoflurane mixture accordingly.

\section{Part I: Preparation for EMG implantation Timing $\sim 30$ min}

5 Follow option A to build a head-mounted connector for rats and option B to implant the amplifier and wireless transmitter of a wireless EMG system into monkeys.

(A) Preparation of rats for EMG implantation

(i) Position the rat in a ventral decubitus position to access the skull. Make a $1.5-\mathrm{cm}$ skin and scalp incision on the head of the animal. Use retractors to pull the scalp to the sides and see the cranial sutures.

(ii) Scratch the connective tissues and periosteum to expose the skull and thus facilitate placement of screws. Using the tip of the scalpel, scratch the surface of the skull to improve the adherence of the dental cement.

(iii) Using a hand drill, drill three holes through the whole thickness of the skull (tip diameter: $0.9 \mathrm{~mm}$ ).

! CAUTION Use a drill with short tips (length $<1.5 \mathrm{~mm}$ ) to avoid brain damage. The skull must be completely perforated to ensure the best adherence between the screws and the skull.

(iv) Insert a screw (length: $3.0 \mathrm{~mm} /$ diameter: $1.0 \mathrm{~mm}$ ) into each hole and turn it 2-2.5 times with a screwdriver (depth $=$ thickness of the skull $=\sim 1 \mathrm{~mm}$ ) (Fig. 3a).

(v) Perform a skin incision on the animal's back. Tunnel EMG and/or stimulation wires (in the case of wired EMG recordings and/or spinal cord stimulation) from the head incision to the back incision, using a straight hemostatic clamp (Fig. 3b).

(B) Preparation of monkeys for EMG implantation

(i) Make a skin incision in the abdomen and insert the implantable EMG amplifier and transmitter.

Part I: Implantation of EMG electrodes into muscles Timing $\sim 30$ min per muscle

6 Position the rat or monkey in a dorsal position to ease access to the leg muscles. Perform a skin incision to access the targeted muscle, while keeping the size of the opening as small as possible to facilitate recovery.

7 Carefully separate the skin and the fascia covering the muscle, using a dental plugger with a rounded tip. Perform an incision of the fascia to maximize the view of the targeted muscle (Fig. 3c).

8 Use thin hemostatic clamps to pull the wires to the targeted muscle through the skin incisions (Fig. 3d). We recommend dissecting all the muscles first and then passing all the wires before initiating the implantation. In rats, it is recommended to pass the wires from the head-mounted connector along the sides of the animal to avoid cross-talk during stimulation and inflammation caused by friction with the vertebras.

9 Use a bipolar probe to locate the motor endplate of the muscle, i.e., the region of the muscle that is the most sensitive to electrical stimulation (Fig. 3e). Probe the muscle throughout the exposed surface using $1-\mathrm{Hz}$ stimulation at amplitudes between 0.5 and $10 \mathrm{~V}$ and with a $300-500 \mu$ s pulse width. 
$\triangle$ CRITICAL STEP Observe carefully the amount of muscle contraction in response to the stimulation and mark the spot at which the largest muscle contractions are observed.

10 Bend two needles to form curved $U$ shapes that will be used to insert two wires into each targeted muscle (Fig. 3f). Insert both needles into the targeted muscle and push the sharp ends out of the muscle (Fig. 3g). The middle of the needle (where the bending occurs) should be located where the most responsive part of the muscle was identified by electrical stimulation. The needles should be 2-3 $\mathrm{mm}$ apart in rats and $\sim 1 \mathrm{~cm}$ apart in monkeys, depending on the size of the targeted muscle.

! CAUTION The diameter of the needle should be as small as possible to avoid muscle damage. $\triangle$ CRITICAL STEP The degree of bending of the needle should be adjusted to ensure that the middle of the needle terminates in the center of the muscle. This will be used as the point of reference for placing the EMG electrode.

11 For both rats and monkeys, remove $\sim 0.5 \mathrm{~mm}$ of insulation from the silicone-coated stainless-steel wires to create a recording electrode (Fig. 3). We recommend preparing the electrodes under a microscope before the surgery.

! CAUTION The electrodes should terminate in the center of the muscle, where the stimulation hot spot was found. It is therefore important to place the electrode at a distance from the tip of the wire that is sufficient to ensure its location within the center of the muscle, in correspondence with the middle of the needle (see Step 16, roughly $1 \mathrm{~cm}$ in rats and $2 \mathrm{~cm}$ in monkeys).

12 Insert the wires into the needles from the sharp endings and pull them from the other side to ensure that the electrode site (where the insulation has been removed) is located at the middle of the bent needle (Fig. 3h).

13 Holding the wires on the sharp ending side, pull the needles out of the muscle, leaving the two wires inside the muscle. If the procedure has been performed properly, the EMG recording site (uninsulated part of the wire) should be located at the stimulation hot spot, i.e., the part of the muscle that is most responsive to electrical stimulation, with the highest number of muscle fibers.

14 In rats (and monkeys, if you are using a wired EMG system), deliver electrical stimulation (for both animals: $1 \mathrm{~Hz}, 500 \mu$ s pulse width, 1-10 V) through the electrodes to locate the strongest responses and thus fine-tune the position of the electrodes (wires).

15 Secure the wires at the entrance and exit of the muscle with sutures (Ethilon 4.0, Fig. 3i). The caudal knot needs to secure the wire close to the exit of the muscle, as the wire tends to move down during movement. Instead, it is important to leave a 1 - to $2-\mathrm{mm}$ distance between the rostral knot and the entrance of the muscle to avoid damage during muscle contraction. Cut the unused wires endings and isolate the tips electrically using liquid plastic welding.

$\triangle$ CRITICAL STEP The knots must lie flat over the muscle. In rats, the extremities of the knot must be short $(1 \mathrm{~mm})$ to avoid irritating the skin and tissues during movement. In monkeys, the extremities of the knot must be at least 3-4 mm to avoid retraction of the wires within the muscle.

16 Use forceps or dissection scissors to form a pocket rostral to the entry point of the wires. Create a stress-release loop and insert it within the pocket to allow for mechanical compliance during movement execution.

17 Suture the fascia and skin openings using U-shaped sutures (Fig. 3j).

18 Repeat Steps 7-17 for each of the targeted muscles. When all muscles are implanted, remove $1 \mathrm{~cm}$ of silicon-coating insulation from the reference wire and place it under the skin, far from the recording sites (e.g., close to the right shoulder in rats).

\section{Part I: Preparation for insertion of the spinal implant Timing 30 min}

19 Position the animal in a prone position and follow option A for rats and option B for monkeys.

(A) Preparation of rats for insertion of the spinal implant

(i) Palpate the vertebral column through the skin to identify the L6 vertebra crest. Its spinous process is substantially larger than the adjacent sacral vertebrae. Use this location as a landmark to locate the T9, T10 and T11 vertebrae.

(ii) Perform a vertical skin incision along the vertebral column, from T11 to the L6 vertebra (Fig. 4).

(iii) Perform incisions of the muscular fascia and detach the muscles along each side of the spinal processes and laminae from T13 to L6 in rats.

$\triangle$ CRITICAL STEP Double-check that the vertebrae were properly identified before proceeding further. 
(iv) In rats, form a subcutaneous 'pocket' laterally at the level of the L4-L6 vertebrae to be able to cover the implant interconnect and dental cement. Pass the connector and the wires connected to the spinal implant within the muscles at the level of the L4/L5 segments (Fig. 4).

(v) Perform five partial laminectomies: between L3 and L4 to prepare the entry point of the spinal implant; between L2 and L3, L1 and L2 and T13 and L1 to check for the positions of active sites of the spinal implant; and between T12 and T13 to pull out the tip of the spinal implant.

! CAUTION In rats, when performing laminectomies, use Adson forceps with teeth to pull up the vertebra and avoid spinal cord damage.

! CAUTION Keep the exposed dura mater hydrated by using gauze or cotton pellets soaked in saline.

$\triangle$ CRITICAL STEP It is important to remove a minimum of bone and joints to avoid the development of spinal deformities. In principle, by removing the posterior longitudinal ligament between two spinal processes and then by performing a yellow ligament flavectomy with a rim of laminae removal, the access should be large enough to insert the implant.

(vi) Retract the muscles from the spinal processes of vertebrae L4, L5 and L6. Clean and dry the vertebrae L4 and L5 with cotton pellets.

(vii) Use a hand drill (tip diameter $=0.6 \mathrm{~mm}$ in rats) to make two holes in the laminae of vertebrae L4 and L5 on both sides of the spinal processes.

$\triangle$ CRITICAL STEP Pull the vertebrae up during drilling to avoid spinal cord damage.

(viii) Insert stainless-steel self-tapping screws (diameter $0.7 \mathrm{~mm}$ ) into each hole and perform two full rotations with a screwdriver.

! CAUTION Check the stability of the screws carefully.

(B) Preparation of monkeys for insertion of the spinal implant

(i) Place the animal in a stereotaxic frame and add soft pads below the thorax to position the vertebral column horizontally.

! CAUTION The abdomen should not be compressed by the pads, to favor the venous return.

(ii) Identify the L5, L4, L3, L2 and L1 spinous processes by palpation, using the L7 vertebra as a landmark. The transition between the S1 and L7 spinous processes can be clearly identified, as the L7 vertebra is comparatively larger than the adjacent sacral vertebra. The L6 spinous process is located between the iliac crests. Generally, the lumbar vertebrae spinous processes have similar size.

? TROUBLESHOOTING

(iii) Perform a skin incision along the vertebral column, from T12 to the L6 vertebrae.

(iv) Perform incisions into the muscular fascia and detach the muscles along each side of the spinal processes and laminae, from L1 to L6.

$\triangle$ CRITICAL STEP Double-check that the vertebrae were properly identified before proceeding further.

(v) Perform five partial laminectomies: between L4 and L5 to prepare the entry point of the spinal implant; between L3, L1 and L2, and T12 and L1 to check for the position of active sites and the mediolateral orientation of the spinal implant (remove only the posterior longitudinal ligament) and between T12 and L1 - to remove the tip of the spinal implant. ! CAUTION Keep the exposed dura mater hydrated by using gauze or cotton pellets soaked in saline.

$\triangle$ CRITICAL STEP It is important to remove a minimum of bone and joints to avoid the development of spinal deformities. In principle, by removing the posterior longitudinal ligament between two spinal processes and then by performing a yellow ligament flavectomy with a rim of laminae removal, the access should be large enough to insert the implant.

\section{Part I: Implantation of the spinal implant Timing 20 min}

20 Follow option A for rats and option B for monkeys.

(A) Implantation of the spinal implant in rats

(i) Tunnel the head-mounted connector of the implant through the hole that was previously made in the trunk muscles (created in Step 19A(iv)). Then, using a hemostat, tunnel the connector below the skin, up to the head of the animal. 
(ii) Position the spinal implant over the vertebral column to locate the tip over the L5 vertebra. Remove $1 \mathrm{~cm}$ of insulation from the reference wire and place the wire under the skin close to the left shoulder.

(iii) Fold an Ethilon 4.0 suture to create a U shape. Pass the suture under the vertebrae, entering from the most rostral opening and exiting at the level of the L4-L5 laminectomy (Fig. 4).

(B) Implantation of the spinal implant in monkeys

(i) Position the tip of the spinal implant over the L5 vertebra.

(ii) Pass a silicone guide that presents the same width as the spinal implant along the midline of the spinal cord. Attach a suture at the extremity of the guide and create a small loop, within which the spinal implant will be inserted, and then folded over with the help of a forceps.

$\triangle$ CRITICAL STEP Use the laminectomies at each step of the insertion procedure to verify that the tip of the suture or guide follows a straight path along the midline of the spinal cord.

21 Fold the tip of the spinal implant within the U-shaped suture.

! CAUTION Leave enough space between the bent part of spinal implant and the first electrode to avoid damaging the contacts.

22 Pull the suture while guiding the spinal implant to position the implant in front of the opening of the spinal canal in a flat, horizontal orientation.

$\triangle$ CRITICAL STEP Hold the tip of the implant with forceps to keep the folded part of implant under tension while inserting it into the spinal canal.

23 Gently pull the implant under the vertebra. Use the laminectomies to monitor the orientation and location of the implant (Fig. 4). Make sure that the most rostral electrode contact is located below the T13 vertebra in rats and the L1 vertebra in monkeys.

! CAUTION Apply saline to all the laminectomy sites to facilitate the insertion of the implant. Ensure that the medial electrode sites remain over the midline of the spinal cord.

Part I: Intraoperative electrophysiology and finalization of the implantation procedure for the spinal implant $\bigcirc$ Timing $\sim 25$ min-1 h

24 Connect the EMG electrodes and the spinal implant to the electrophysiological setup. In the case of head-mounted connectors, connect the EMG wires to a multichannel low-impedance differential amplifier (e.g., a PZ5 amplifier) and the wires from the spinal implant to a multichannel stimulator (e.g., an IZ2H stimulator). Both the amplifier and the stimulator should in turn be connected to a real-time data acquisition and control system (e.g., an RZ2 BioAmp processor). When using a fully implantable electrical stimulator, clamp the electrode connector with crocodile clips during intraoperative procedures. At the end of the procedure, connect the electrode to the implantable stimulator.

25 Display and store stimulation-pulse-triggered EMG signals within a 50-ms window.

26 Set the stimulator to current mode (voltage mode can also be used, but, for various reasons, it is not preferred $^{41}$ ). Set the stimulation frequency to $0.5 \mathrm{~Hz}$. In general, expect a current range from 0 to $600 \mu \mathrm{A}$ in rats and 0 to $5 \mathrm{~mA}$ in monkeys, using a $200-\mu$ s pulse-width in rats and a $300-\mu$ s pulsewidth in monkeys.

\section{? TROUBLESHOOTING}

27 Stimulate the most rostral sites at increasing intensities to verify that the motor responses elicited in the IL muscle emerge at a lower threshold than the responses in other leg muscles. Stimulation of the most rostral lumbar segments induces an isolated hip flexion after each stimulation pulse, provided the intensity of stimulation remains close to the motor threshold.

! CAUTION High current levels may induce strong movements in the animal, with coactivation of many muscles.

\section{? TROUBLESHOOTING}

28 Stimulate the most caudal sites at increasing intensities to verify that the motor responses elicited in the medial gastrocnemius muscle emerge at a lower threshold than the responses in other leg muscles. Expect a current amplitude ranging from 100 to $300 \mu \mathrm{A}$ in rats and 1 to $2 \mathrm{~mA}$ in monkeys, using a $200-\mu$ s pulse-width in rats and a $300-\mu$ s pulse-width in monkeys. Stimulation of this region should induce an isolated ankle plantar flexion after each stimulation pulse.

$\triangle$ CRITICAL STEP Do not confuse ankle plantar flexion movements with knee flexion movements, which indicate recruitment of the knee flexors, rather than ankle extensor muscles.

? TROUBLESHOOTING 
29 Slide the implant to fine-tune the longitudinal position of the electrode contacts. For each position, repeat Steps 27 and 28 until optimal conditions are met.

? TROUBLESHOOTING

30 To ensure that the position of the spinal implant in the mediolateral direction is adequate, stimulate through the lateral sites. Ensure that the muscles of the leg ipsilateral to the stimulation site are recruited at a markedly lower current level compared with the muscles of the contralateral leg. Adjust the position of the implant, using the openings provided by the laminectomies at the various spinal levels.

! CAUTION Mediolateral movements of the spinal implant may also modify the longitudinal positioning, especially in rats. Repeat Steps 27 and 28, if necessary.

$\triangle$ CRITICAL STEP Make sure that the animal is not in a twisted position, as this may influence the final location of the implant. In the ideal position, there will be no rotation in the vertebral column, and the lordosis and kyphosis will exhibit natural curvature.

31 To finalize implantation, follow option A for rats or option B for monkeys

(A) Finalization of implantation in rats

(i) Prepare a base for the orthosis by placing dental cement around the screws at the L4/L5 vertebrae.

(ii) Place the connector of the implant between the screws located at vertebra L5. Using dental cement, form an orthosis around the connector.

! CAUTION Dental cement should never come into contact with the implant or the connector.

! CAUTION Carefully verify the angle and location of the screws with respect to the vertebrae body to avoid damaging the spinal cord with the tips of the screws.

(iii) Finalize the implantation of the head-mounted connector.

$\triangle$ CRITICAL STEP Form stress-releasing loops with wires and place them within the prepared pockets (Steps 19) at the level of the belly to avoid mechanical stress on the implant.

(B) Finalization of implantation in monkeys

(i) Secure the titanium orthosis at the L4/L5 junction, using titanium self-drilling screws that have a length of $6 \mathrm{~mm}$ and a diameter of $1.5 \mathrm{~mm}$.

(ii) Perform a longitudinal skin incision along the abdomen and then prepare a pouch between the intercostal muscles in order to insert the implantable stimulator. Then, tunnel the wires connected to the spinal implant to the abdomen and connect them to the stimulator. For more information, follow standard Medtronic procedures for implantation of pulse generators.

$\triangle$ CRITICAL STEP Make sure to create stress-releasing loops between the spinal implant and the stimulator to avoid mechanical pulling of the implant in the first days after surgery. Suture the stimulator to the fascia to avoid migration.

32 Close and suture the incisions in three layers (fascia, subcutaneous layer and skin)

! CAUTION Use internal skin stitches to form clean skin scars and reduce the risk of infection and pain to the animals.

\section{Part I: Post-surgical care Timing 3-7 d}

33 Immediately after surgery, follow option A for rats or option B for monkeys.

(A) Post-surgical care of rats

(i) Place the rat in a warm incubator until it is fully awake.

(ii) Perform a s.c. injection of 3-5 $\mathrm{ml}$ of warm Ringer's solution.

(B) Post-surgical care of monkeys

(i) Place the animal in a dedicated recovery cage with soft bedding and illuminate the animal with an IR warming light until the animal is fully awake.

(ii) Provide the animal with fruit juice and/or water until fully awake, then feed the animal with its usual daily food intake quantity.

34 After the animal wakes up, inject buprenorphine $(0.01-0.05 \mathrm{mg} / \mathrm{kg}$, s.c.) to provide postoperative analgesia. Follow up with same dosage once a day for 5-7 d.

35 For the next 5-7 d, inject antibiotics regularly. Amoxicillin (Clamoxyl) is an example of a suitable antibiotic that can be used at the following doses: $0.5 \mathrm{ml} / \mathrm{kg}$ two times per day in rats, $30 \mathrm{mg} / \mathrm{kg}$ once a day in monkeys). 
Part II: Animal preparation and electrophysiological setup for post-surgical selection of optimal electrode configurations Timing $\sim 1 \mathrm{~h}$

36 Sedate the animal with ketamine $(10 \mathrm{mg} / \mathrm{kg}$, i.m. injection).

37 Place the animal in a position that allows movement of the legs. For example, the animal can be suspended in the air in a custom-made harness or jacket.

38 Set up the EMG amplification and data acquisition system. In rats, connect the head-mounted connector receiving the EMG wires to a multichannel low-impedance differential amplifier (e.g., a PZ5 amplifier). Connect the amplifier to a specialized data acquisition system (e.g., an RZ2 BioAmp processor). In monkeys, connect a wireless headstage to the head- mounted connector (e.g., a W16 16-channel wireless headstage). Connect the radiofrequency receiver to a data acquisition system, using analog inputs (e.g., a DAQ board from National Instruments or an RZ2 BioAmp processor).

39 Set up the spinal implant. In rats, connect the head plug receiving the wires from the spinal implant to a multichannel stimulator (e.g., an IZ2H stimulator) controlled by a computer or real-time processor (e.g., an RZ2 BioAmp processor). In monkeys, establish the communication with the IPG (e.g., an Activa RC implantable pulse stimulator).

40 (Optional) Set up a video recording or motion-capture system to record the movements that will be induced by EES during the following step.

\section{Part II: Characterization of the spatial selectivity of the implant Timing $\sim \mathbf{2} \mathrm{h}$}

41 Follow a procedure similar to that described in Steps 27 and 28. Select an electrode contact and deliver single, bipolar and charge-balanced electrical pulses (200- $\mu$ s pulse width in rats, $300-\mu$ s pulse width in monkeys) at a frequency of $0.5 \mathrm{~Hz}$ to avoid interactions between successive pulses. Set the selected electrode contact as the cathode (negative polarity). In monkeys, the anode (positive polarity) can be the case of the IPG or one of the contacts of the spinal implant for multipolar configurations.

42 Manually increase the stimulation amplitude until a motor response is observed in the targeted muscles. A motor potential elicited by EES occurs with a latency of $\sim 3-8 \mathrm{~ms}$ in rats and 5-15 $\mathrm{ms}$ in monkeys after stimulation onset. Write down the intensity corresponding to the motor threshold for the targeted muscle group.

43 Increase the intensity of EES until saturating the amplitude of the motor responses for all the muscles. Write down the stimulation amplitude that reaches saturation.

! CAUTION Spinal cord stimulation at high amplitudes can induce very strong responses in leg muscles. Do not exceed amplitude limits that can create harm to the animal due to sudden strong, whole-limb movements.

44 Record EMG signals while systematically ramping up the stimulation amplitude from 0.9 times the motor threshold found at Step 42 to the saturation amplitude found at Step 43.

$\triangle$ CRITICAL STEP Set at least four stimulation pulses per amplitude (for averaging) and ten amplitude steps for the investigated range. Examine the muscle recruitment order while performing the recording and store the raw data for future analysis.

45 Repeat Steps 41-44 for each electrode of the spinal implant, until the muscle responses evoked by each of the electrode contacts are recorded.

46 Due to slight offsets in the location of the implant and intrinsic variability between animals, the specificity obtained with monopolar stimulation might not be sufficient. In this scenario, set up multipolar configurations, as this may increase the specificity. For example, when stimulations applied through the lateral electrodes induce responses in muscles from both sides, it is possible to shield the side of the spinal cord that should not be activated. To do this, place a cathode on the targeted side, and one or several anodes on the midline and/or on the opposite side. This configuration should steer the activating field toward the desired side. A similar principle can be used to increase rostrocaudal selectivity. For example, if the IL muscle (most rostral motor pools) is not specifically recruited by the most rostral electrodes, place the cathode on the most rostral electrode and one or several anodes on the electrodes caudal to the cathode. For each of the selected multipolar configurations, repeat Steps 41-44.

? TROUBLESHOOTING

47 When all the recordings are completed, implement the local procedures defined for animal awakening and post-sedation care (Step 33).

48 Calculate the recruitment curves from the data obtained in Steps 41-46. Recruitment curves indicate the normalized level of activation of each muscle in response to single electrical pulses of increasing 
amplitude (Fig. 5). Normalize the responses by using the maximum across all stimulation amplitudes and all stimulation sites. If required, also translate these motor responses into spatial maps of motoneuron pool activation (Fig. 5). From the recruitment curves, identify a functional range of stimulation amplitudes over which only the muscles activated at the lowest thresholds are substantially recruited. Compute the spatial maps of motoneuron activation corresponding to this functional range, and use them to define the spatial specificity of each electrode configuration.

49 Use the analyses from Step 48 to determine the electrode configurations that yield the highest degree of activation in the spinal segments responsible for the flexion of the leg, especially hip flexion (L1-L2 in both rats and monkeys), while restricting responses to one side of the spinal cord over a wide range of amplitudes. Select this configuration to promote a global flexion of the leg. Similarly, determine the electrode configurations that yield the highest degree of activation in the spinal segments responsible for extension of the leg, especially ankle plantar flexion (L4-L6 in rats, L6-L7 in monkeys), while restricting responses to one side of the spinal cord over a wide range of amplitudes. Select this configuration to promote a global extension of the leg.

\section{Part III: Determination of stimulation patterns $\bigcirc$ Timng 3-4 h per iteration; to be repeated every day for 1-2 weeks}

$\triangle$ CRITICAL A real-time monitoring system must be established and used that reliably detects or predicts 'foot off and 'foot strike' events (Fig. 7). This system can be based on kinematic recordings when the animal retains residual limb movements (as described in this section). If this is not possible, an alternative is to decode these events from neural signals, using intracortical microelectrode arrays.

50 Position the animal on a treadmill located at the center of the motion-capture system for the detection of gait events and recording leg kinematics (Fig. 1).

51 Start by programming, for each leg independently, the onset of flexion and extension stimulation bursts at the 'foot off and 'foot strike' events, respectively.

52 Predetermine the duration of each burst in order to cover at least half of the swing phase for the flexion site and the entire duration of the stance phase for the extension site. Alternatively, the duration of each burst can be determined by additional gait events, which can be used to terminate the bursts, i.e., mid-swing.

$\triangle$ CRITICAL STEP After severe lesion, the lack of excitability may compromise the ability of the spinal cord to activate muscles with sensory feedback, as for example, weight-bearing input during the stance phase. In this condition, the absence of stimulation during a certain period of the stance phase may lead to the collapse of the animals. Always ensure that the extension stimulation provides support when the animal uses its leg to support its weight.

53 Select initial amplitudes and frequencies. Select a frequency of about $40 \mathrm{~Hz}$ for all electrode configurations. For each electrode configuration, select an amplitude $\sim 1.5$ times the motor threshold obtained during the recruitment curves (Step 48).

54 Test the spatiotemporal neuromodulation protocols with the initial set of parameters defined in Steps 51-53. Adjust the stimulation amplitudes based on the kinematic readout and modulation of EMG activity. Each electrode configuration should have a substantial effect on the targeted muscle group without loss of muscle specificity.

! CAUTION High stimulation amplitudes may induce a blockage of one or both legs; slowly adapt the amplitudes to avoid such issues.

? TROUBLESHOOTING

55 Refine the stimulation timing empirically. Anticipate or delay the onset of each stimulation burst and observe changes in kinematics and EMG activity. Kinematic effects can be quantified by looking at key variables such as step height or stride length. Similarly, extend or reduce the duration of each stimulation burst and examine the effect on kinematics and EMG activity. Select the timings that maximize the step height during the swing phase and whole-limb extension during the stance phase.

56 Refine the stimulation amplitudes and frequencies, knowing that increasing amplitude or frequency within a certain range increases the size of the kinematic and kinetic effects of the stimulation (Fig. 8). Adjust the amplitudes and frequencies by following the procedure described in Step 55. Select the minimal amplitude for a defined frequency that maximizes the step height during the swing phase and whole-limb extension during the stance phase.

! CAUTION To increase the level of activation of muscles, it is preferable to modulate the frequency of stimulation (recruitment of the same fiber more frequently), as increases in amplitude may lead to the activation of additional, nondesired muscles (recruitment of additional fibers). 

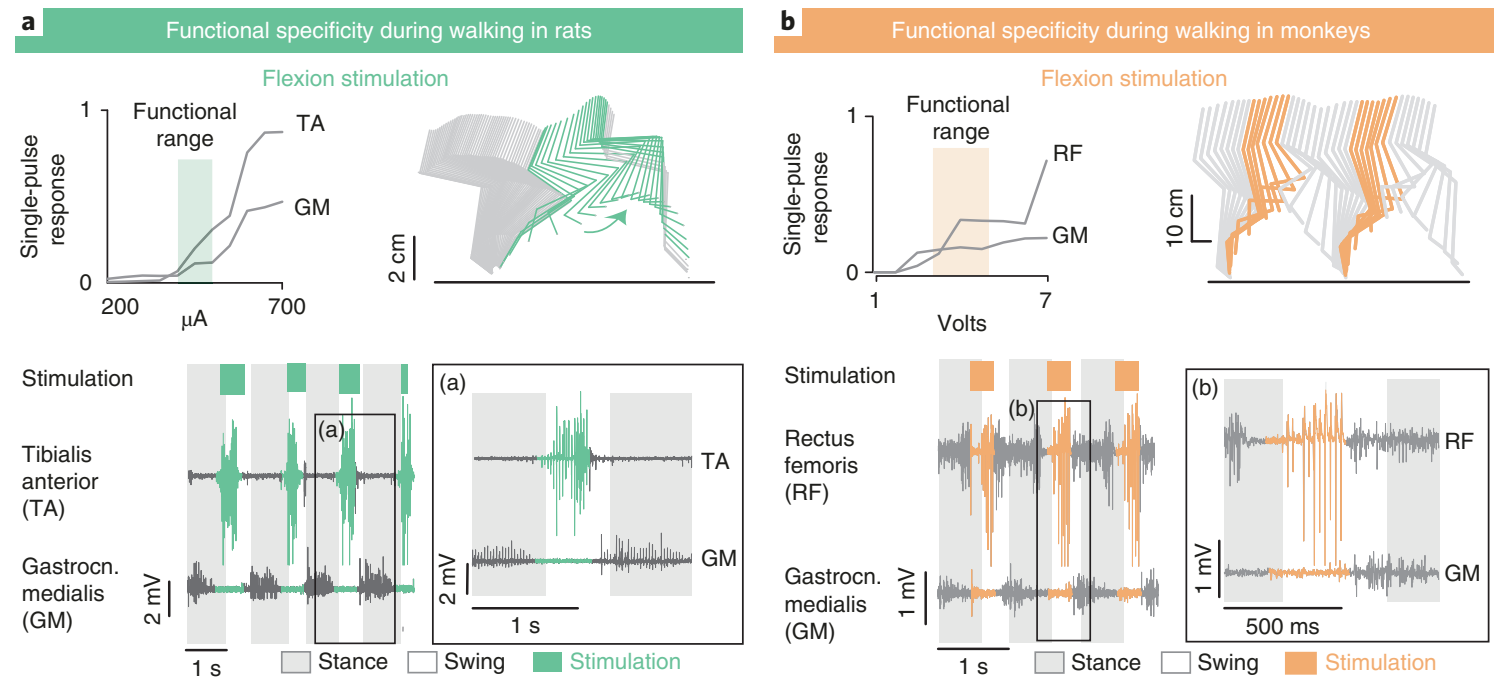

Fig. 9 | Enhanced functional specificity of spatiotemporal neuromodulation during walking. a,b, For both rats (a) and monkeys (b), the functional specificity of different stimulation sites (here shown for the flexion stimulation site only) is enhanced during walking, compared with the effects during single-pulse stimulation. a, Recruitment curves, on the left, showing that both flexor (tibialis anterior) and extensor (gastrocnemius medialis) muscles are recruited by single-pulse stimulation in sedated animals, even within the range normally used during behavioral experiments (functional range). When applying spatiotemporal neuromodulation of this specific stimulation site during the swing phase (bottom), the flexor muscles are exclusively recruited, whereas the extensor muscles are silenced (EMG traces shown). This enhanced specificity is due to the interactions between the stimulation and the incoming gait-related proprioceptive inputs and descending cortical inputs within the lumbar spinal cord. a (inset), A zoomed view of the EMG activity, in which suppression of extensor muscles and enhanced activity of the flexor muscles are visible during the swing phase. Periods in which stimulation is ON are shown in green in the EMG traces and in the stick diagrams. b. Corresponding effects and data for Rhesus monkeys. EMGs of the RF and GM are shown. In this example, the stimulation active during the swing phase enhances the activity of the RF while showing no influence on the GM muscles, despite the clear cross-talk observed in the recruitment curve. $\mathbf{b}$ (inset), A zoomed view of the EMG activity in the swing phase. Periods in which stimulation is ON are shown in orange in the EMG traces and in the stick diagrams. a adapted with permission from Wenger et al. ${ }^{20}$, Springer Nature Limited; $\mathbf{b}$ adapted with permission from Capogrosso et al. $^{8}$, Springer Nature Limited. All animal experiments depicted in this figure were approved by the local authorities.

57 Repeat Steps 51-56 until an optimal set of parameters is found.

! CAUTION Due to spontaneous and training-induced motor recovery, the spatial and temporal structure of stimulation protocols may need to be adapted regularly.

\section{Troubleshooting}

Troubleshooting advice can be found in Table 1.

\section{Table 1 | Troubleshooting table}

Step Problem Possible reason Solution

19 Difficulty in the identification of Anatomical variability the $\mathrm{L} 7$ vertebra in monkeys motor response within the stimulator ranges
Identification of the L7 vertebra by skin palpation can be difficult. The anatomical variability, particularly of the $\mathrm{S} 1$ spinal process, can lead to confusion. In such cases, rely on the similarity between the L6, L5 and L4 spinal processes. These vertebrae are substantially larger than the $L 7$ vertebra. They have a long flat process similar in size and shape to L7. The L7 vertebra presents a flat process but is substantially smaller than the L6 vertebra. The S1 vertebra can either be impossible to feel or feel like a spiky process. In addition, note that the L7 and L6 vertebrae are close to the iliac crest

Check cables and connections

High electrode impedance
If the electrode impedance is too high, the requested current level may not be reached by the stimulator because of limited voltage compliances. In such cases, the current value indicated by the stimulation hardware/software is not correct. Increase the pulse width to $500 \mu$ s to reduce the current threshold of the axons. If no responses are shown at $500 \mu \mathrm{s}$, consider changing the spinal implant or label the electrode as nonfunctional

Table continued 
Table 1 (continued)

\section{Step Problem}

27 The most rostral electrode does not recruit hip flexion muscles

\section{Possible reason}

Implant is too caudal

Implant is too rostral

The most caudal electrode does not recruit ankle extensors

Implant is too caudal

Implant is too rostral

29 It is impossible to find a longitudinal placement of the implant at which both hip flexors and ankle extensors can be recruited in isolation
Implant is short compared with the size of the animal's spinal cord

The spinal cord excitability changes after injury

Implant was poorly placed during surgery or has moved over the mediolateral axis
$54 \quad$ Selected amplitude ranges do not lead to functional responses predicted during the postoperative identification of optimal electrode configurations
Thresholds of axons change according to the relative position of the spinal cord

Descending modulatory drive or $\mathrm{SCl}$ changed the responses of the spinal circuits to electrical stimulation

\section{Solution}

If the hip flexor muscles (e.g., IL) are recruited together with strong quadriceps twitches or knee flexors (such as the semitendinosus), move the implant to a more rostral location

If there is no hip flexor recruited, but strong twitches of back muscles are present, it means that the implant is too rostral. Move the implant to a more caudal location

If tail twitches are evident, but no simultaneous activation of the gastrocnemius muscle or other ankle extensors is present, the implant is too caudal. Move the implant to a more rostral location

If movement of the ankle extensors is mixed with twitches of the knee flexors or extensors, it means that the implant is too rostral. Move the implant to a more caudal location

If the optimization of the implant along the rostral and caudal directions is not sufficient to support independent recruitment of hip flexors and ankle extensors, it implies that the implant is too short compared with the size of the spinal cord. Either inappropriate design of the implant or interanimal variability can cause this. We suggest using a rostral placement of the implant to ensure good selectivity of the hip flexor. Indeed, hip flexors are critical to initiating the swing phase. Weight bearing can be potentiated using knee extensors instead of ankle extensors

The response to EES evolves between the intact state and the period of recovery after the $\mathrm{SCI}$. Typically, the reflex responses may be larger and thus triggered at lower thresholds than in the intact state. For example, flexor muscles of the knee might be activated by nearly all the electrodes of the spinal implant, which may lead to confusion regarding the anatomical placement of the spinal implant. Keep this possibility in mind and focus on obtaining the best possible specificity over the activation of hip flexion and ankle extension

The use of a transversal multipolar configuration can help to increase the specificity of the stimulation. By injecting anodal current over the midline or through the electrode contralateral to the targeted region, it is possible to steer the stimulating cathodal field toward one side of the spinal cord. For example, it is possible to increase the ability to target the left hip flexors when placing an anode on the right side at the same level as the cathode

The posture of the animal alters the distance between the spinal cord and the spinal implant, which results in threshold changes between the lying position and the upright position. This change should affect only the level of current needed to elicit responses. Therefore, it should be sufficient to increase the current amplitude, compared with that of the post-operative tests, to obtain meaningful responses

In animals with chronic $\mathrm{SCl}$, the excitability of proprioceptive circuits changes substantially as compared with that of the healthy state. If necessary, repeat the postoperative optimization of optimal electrode configurations to identify new electrode configurations better adapted for the new state of the spinal circuits. Alternatively, repeat the optimization of the burst-timing procedures, which critically affect the functional outcome of stimulation bursts because of the different modulation of spinal circuits during the gait cycle (see also Fig. 7)

Part I

Steps 1-4, animal preparation for surgery: $\sim 30 \mathrm{~min}$

Step 5, preparation for EMG implantation: $\sim 30 \mathrm{~min}$

Steps 6-18, implantation of chronic EMG leads: $\sim 30$ min per implanted muscle

Step 19, preparation of the spinal cord for electrode implantation: $\sim 30 \mathrm{~min}$

Steps 20-23, implantation of the spinal implant: $\sim 20 \mathrm{~min}$

Steps 24-32, fine-tuning of electrode positioning: $\sim 25 \mathrm{~min}-1 \mathrm{~h}$

Steps 33-35, post-surgical treatment, standard care procedures: $\sim 3-7 \mathrm{~d}$. 


\section{Part II}

Steps 36-40, preparation of the system and animal for post-surgical optimization of electrode configuration: $\sim 1 \mathrm{~h}$.

Steps 41-49, selection of optimal electrode configuration: $\sim 2 \mathrm{~h}$

Part III

Steps $50-57$, selection of optimal timing of stimulation bursts: $3-4 \mathrm{~h}$ per procedure, to be repeated until the desired motor output is reached.

Spinal implant positioning and selection of optimal electrode configurations

Post-operative mapping is a critical step for the selection of the electrode configurations that will be used during locomotion. During the procedure that we describe, the anatomical landmarks should ensure a positioning of the implant in rostral sites that elicit recruitment of the most rostrally innervated leg muscles and caudal sites that recruit the most caudally innervated leg muscles (Fig. 2). Figure 5 shows examples of motor responses in rats and monkeys. For each leg, a 'flexion site' and an 'extension site' are selected to maximize the specificity for either flexor muscles innervated from motoneurons located in rostral lumbar segments (e.g., IL) or extensor muscles innervated from motoneurons located in caudal lumbar segments (e.g., gastrocnemius). Surgical positioning should also ensure a left/right specificity. In the ideal case, symmetrical sites on the spinal implant would elicit similar motor responses in the muscles of the left and right legs. However, this situation is rarely encountered. For example, in Fig. 5, the optimal electrode for the right flexion of the monkey was selected as the most rostral medial contact on the spinal implant. The tip of the implant has slightly shifted toward the right side of the spinal cord in this animal. Consequently, the midline electrode became selective for rostral muscles of the right leg. By contrast, the most caudal electrode on the right was selected ${ }^{11,19,20}$ as an 'extension site', as expected from the surgical placement. As shown in both rats and monkeys, the representation of the EMG responses induced by single pulses of EES as spatial maps of spinal segment activation provides a good readout of the spinal roots that are recruited by a given electrode configuration.

\section{Modulation of leg kinematics}

During steady-state walking conditions in either injured or intact animals, adjusting the stimulation amplitude and frequency can lead to robust modulations of kinematic variables. In Fig. 9, we show examples of kinematics and EMG activities in rats with SCI and in intact monkeys during locomotion on a treadmill. Modulation of stimulation frequency or amplitude led to sizeable variations in the kinematic variables and EMG activities associated with flexion or extension, depending on the tuned electrode configuration ${ }^{8,11,19,20}$. For example, increases in amplitude (shown in rats) or frequency (shown in monkeys) of the flexion site elicited a proportional enhancement of the step height. Changes in kinematics tend to be more prominent during modulation of the flexion sites, as the biomechanical constraints due to the loading of the leg during the stance phase limit the modulation of the kinematics. Therefore, variables correlating with the modulation of extension are captured in ground reaction forces (shown in rats) or EMG activity of extensor muscles (shown in monkeys). Importantly, these stimulation parameters (amplitude and frequency) can also be adjusted in real time via a closed-loop control algorithm that aims to maintain a specific desired kinematic variable, such as step height or stride length, within a certain range ${ }^{11,20}$. For example, this approach can be used to compensate for the decrease in step height that emerges during training due to natural fatigue.

\section{Functional specificity of spatiotemporal neuromodulation}

Despite a proper design of the spinal implants and the careful execution of the procedure described above, a series of factors limit the specificity of EES protocols. These limitations include anatomical constraints, subject-specific anatomical features, implant migration and even electrical failure of the optimal active sites. However, as we explained in the Introduction, the integration of EES pulses together with proprioceptive information and descending inputs at the level of spinal sensorimotor circuits confers a high degree of robustness on spatiotemporal neuromodulation protocols, even when the specificity of electrodes configurations is reduced. For example, Fig. 6 shows an occurrence in which a flexion site induced a pronounced activation of ankle extensors $(\sim 50 \%$ of the targeted flexor muscles). This situation would intuitively lead to co-contraction of both extensors and flexion muscles during locomotion, which would reduce the efficacy of the flexion site. However, as sensory 
inputs and residual descending signals gate the excitatory drive elicited by EES, the effect of EES on extensor-related circuits is limited during swing. Indeed, despite the recruitment of the gastrocnemius muscle by the flexion site during rest, no functional activity was recorded in the gastrocnemius when the flexion electrode was activated during the swing phase. Consequently, the flexion site exhibits a high degree of specificity during gait ${ }^{10,12}$. This result implies that the timing of stimulation is a critical feature that can be used to boost the spatial specificity of the selected sites when the stimulation is applied at the right moment of the gait cycle. Setting up spatiotemporal neuromodulation requires careful adjustment of multiple parameters and involves many steps to fine-tune the stimulation protocols. Here, we have described each of these steps in detail and provided typical solutions to the most commonly encountered problems during the execution of the procedure. Despite this complex tuning procedure, the final stimulation protocols are relatively simple and result in robust, functional movements of the legs, before and after SCI.

The human spinal cord exhibits the same segmental responses to $\mathrm{EES}^{87}$ and spatial distribution of leg motoneurons as those of other mammals ${ }^{18}$. Therefore, use of a system similar to that described here has enabled volitional control of leg muscles in individuals with motor-complete SCI, which allowed them to trigger isolated movements of single joints, depending on the site of $\mathrm{EES}^{4,9}$. This, combined with results obtained using the procedures detailed in the present protocol on rats and monkeys, suggests that spatiotemporal neuromodulation therapies could be used to facilitate movement execution in humans with SCI.

\section{References}

1. Bizzi, E., Giszter, S. F., Loeb, E., Mussa-Ivaldi, F. A. \& Saltiel, P. Modular organization of motor behavior in the frog's spinal cord. Trends Neurosci. 18, 442-446 (1995).

2. Levine, A. J. et al. Identification of a cellular node for motor control pathways. Nat. Neurosci. 17, 586-593 (2014).

3. van den Brand, R. et al. Restoring voluntary control of locomotion after paralyzing spinal cord injury. Science 336, 1182-1185 (2012).

4. Angeli, C. A., Edgerton, V. R., Gerasimenko, Y. P. \& Harkema, S. J. Altering spinal cord excitability enables voluntary movements after chronic complete paralysis in humans. Brain 137, 1394-1409 (2014).

5. Harkema, S. et al. Effect of epidural stimulation of the lumbosacral spinal cord on voluntary movement, standing, and assisted stepping after motor complete paraplegia: a case study. Lancet 377, 1938-1947 (2011).

6. Danner, S. M. et al. Human spinal locomotor control is based on flexibly organized burst generators. Brain 138, 577-588 (2015).

7. Courtine, G. et al. Transformation of nonfunctional spinal circuits into functional states after the loss of brain input. Nat. Neurosci. 12, 1333-1342 (2009).

8. Capogrosso, M. et al. A brain-spine interface alleviating gait deficits after spinal cord injury in primates. Nature 539, 284-288 (2016).

9. Grahn, P. J. et al. Enabling task-specific volitional motor functions via spinal cord neuromodulation in a human with paraplegia. Mayo Clin. Proc. 92, 544-554 (2017).

10. Capogrosso, M. et al. A computational model for epidural electrical stimulation of spinal sensorimotor circuits. J. Neurosci. 33, 19326-19340 (2013).

11. Moraud, E. M. et al. Mechanisms underlying the neuromodulation of spinal circuits for correcting gait and balance deficits after spinal cord injury. Neuron 89, 814-828 (2016).

12. Rattay, F., Minassian, K. \& Dimitrijevic, M. R. Epidural electrical stimulation of posterior structures of the human lumbosacral cord. 2. Quantitative analysis by computer modeling. Spinal Cord 38, 473-489 (2000).

13. Gerasimenko, Y. P. et al. Spinal cord reflexes induced by epidural spinal cord stimulation in normal awake rats. J. Neurosci. Methods 157, 253-263 (2006).

14. Minassian, K. et al. Human lumbar cord circuitries can be activated by extrinsic tonic input to generate locomotor-like activity. Hum. Mov. Sci. 26, 275-295 (2007).

15. Taccola, G., Sayenko, D., Gad, P., Gerasimenko, Y. \& Edgerton, V. R. And yet it moves: recovery of volitional control after spinal cord injury. Prog. Neurobiol. 160, 64-81 (2018).

16. Stein, R. B. \& Capaday, C. The modulation of human reflexes during functional motor tasks. Trends Neurosci. 11, 328-332 (1988).

17. Dy, C. J. et al. Phase-dependent modulation of percutaneously elicited multisegmental muscle responses after spinal cord injury. J. Neurophysiol. 103, 2808-2820 (2010).

18. Sayenko, D. G., Angeli, C., Harkema, S. J., Edgerton, V. R. \& Gerasimenko, Y. P. Neuromodulation of evoked muscle potentials induced by epidural spinal-cord stimulation in paralyzed individuals. J. Neurophysiol. 111, 1088-1099 (2014).

19. Wenger, N. et al. Closed-loop neuromodulation of spinal sensorimotor circuits controls refined locomotion after complete spinal cord injury. Sci. Transl. Med. 6, 255ra133 (2014).

20. Wenger, N. et al. Spatiotemporal neuromodulation therapies engaging muscle synergies improve motor control after spinal cord injury. Nat. Med. 22, 138-145 (2016). 
21. Yakovenko, S., Mushahwar, V., VanderHorst, V., Holstege, G. \& Prochazka, A. Spatiotemporal activation of lumbosacral motoneurons in the locomotor step cycle. J. Neurophysiol. 87, 1542-1553 (2002).

22. Ivanenko, Y. P. et al. Temporal components of the motor patterns expressed by the human spinal cord reflect foot kinematics. J. Neurophysiol. 90, 3555-3565 (2003).

23. Wenger, N. et al. Closed-loop neuromodulation of spinal sensorimotor circuits controls refined locomotion after complete spinal cord injury. Sci. Transl. Med. 6, 255ra133 (2014).

24. Courtine, G. et al. Transformation of nonfunctional spinal circuits into functional states after the loss of brain input. Nat. Neurosci. 12, 1333-1342 (2009).

25. Minev, I. R. et al. Electronic dura mater for long-term multimodal neural interfaces. Science 347, 159-163 (2015).

26. Capogrosso, M. et al. Advantages of soft subdural implants for the delivery of electrochemical neuromodulation therapies to the spinal cord. J. Neural Eng. 15, 026024 (2018).

27. Cheriyan, T. et al. Spinal cord injury models: a review. Spinal Cord 52, 588-595 (2014).

28. Lukovic, D. et al. Complete rat spinal cord transection as a faithful model of spinal cord injury for translational cell transplantation. Sci. Rep. 5, 9640 (2015).

29. Courtine, G. et al. Recovery of supraspinal control of stepping via indirect propriospinal relay connections after spinal cord injury. Nat. Med. 14, 69-74 (2008).

30. Courtine, G. et al. Can experiments in nonhuman primates expedite the translation of treatments for spinal cord injury in humans? Nat. Med. 13, 561-566 (2007).

31. Capogrosso, M. et al. A brain-spine interface alleviating gait deficits after spinal cord injury in primates. Nature 539, 284-288 (2016).

32. Freund, P. et al. Nogo-A-specific antibody treatment enhances sprouting and functional recovery after cervical lesion in adult primates. Nat. Med. 12, 790-792 (2006).

33. Slotkin, J. R. et al. Biodegradable scaffolds promote tissue remodeling and functional improvement in nonhuman primates with acute spinal cord injury. Biomaterials 123, 63-76 (2017).

34. Rosenzweig, E. S. et al. Extensive spontaneous plasticity of corticospinal projections after primate spinal cord injury. Nat. Neurosci. 13, 1505-1510 (2010).

35. Salegio, E. A. et al. A unilateral cervical spinal cord contusion injury model in non-human primates (Macaca mulatta). J. Neurotrauma 33, 439-459 (2016).

36. Reeve, C. \& Reeve, D. The Spinal Cord (Elsevier, New York, 2008).

37. Coburn, B. A theoretical study of epidural electrical stimulation of the spinal cord-Part II: effects on long myelinated fibers. IEEE Trans. Biomed. Eng. 32, 978-986 (1985).

38. Holsheimer, J. Which neuronal elements are activated directly by spinal cord stimulation. Neuromodulation 5, 25-31 (2002).

39. Cuellar, C. A. et al. The role of functional neuroanatomy of the lumbar spinal cord in effect of epidural stimulation. Front. Neuroanat. 11, 82 (2017).

40. Gad, P. et al. Development of a multi-electrode array for spinal cord epidural stimulation to facilitate stepping and standing after a complete spinal cord injury in adult rats. J. Neuroeng. Rehabil. 10, 2 (2013).

41. Merrill, D. R., Bikson, M. \& Jefferys, J. G. Electrical stimulation of excitable tissue: design of efficacious and safe protocols. J. Neurosci. Methods 141, 171-198 (2005).

42. Cogan, S. F. Neural stimulation and recording electrodes. Annu. Rev. Biomed. Eng. 10, 275-309 (2008).

43. de Jongste, M. J. et al. Stimulation characteristics, complications, and efficacy of spinal cord stimulation systems in patients with refractory angina: a prospective feasibility study. Pacing Clin. Electrophysiol. 17, 1751-1760 (1994).

44. Alo, K. et al. Factors affecting impedance of percutaneous leads in spinal cord stimulation. Neuromodulation 9, 128-135 (2006).

45. Hofstoetter, U. S. et al. Periodic modulation of repetitively elicited monosynaptic reflexes of the human lumbosacral spinal cord. J. Neurophysiol. 114, 400-410 (2015).

46. Segev, I., Fleshman, J. W. Jr. \& Burke, R. E. Computer simulation of group Ia EPSPs using morphologically realistic models of cat a-motoneurons. J. Neurophysiol. 64, 648-660 (1990).

47. Gerasimenko, Y. P. et al. Spinal cord reflexes induced by epidural spinal cord stimulation in normalawakerats. J. Neurosci. Methods. 157, 253-263 (2006).

48. Edgerton, V. R. et al. Training locomotor networks. Brain Res. Rev. 57, 241-254 (2008).

49. Holinski, B. J. et al. Intraspinal microstimulation produces over-ground walking in anesthetized cats. J. Neural Eng. 13, 056016 (2016).

50. Hehenberger, L., Seeber, M. \& Scherer, R. Estimation of gait parameters from EEG source oscillations. IEEE International Conference on Systems, Man, and Cybernetics (SMC) 004182-004187, https://doi.org/10.1109/ SMC.2016.7844888 (2016).

51. Presacco, A., Goodman, R., Forrester, L. \& Contreras-Vidal, J. L. Neural decoding of treadmill walking from noninvasive electroencephalographic signals. J. Neurophysiol. 106, 1875-1887 (2011).

52. Presacco, A., Forrester, L. W. \& Contreras-Vidal, J. L. Decoding intra-limb and inter-limb kinematics during treadmill walking from scalp electroencephalographic (EEG) signals. IEEE Trans. Neural Syst. Rehabil. Eng. 20, 212-219 (2012).

53. Artoni, F. et al. Unidirectional brain to muscle connectivity reveals motor cortex control of leg muscles during stereotyped walking. Neuroimage 159, 403-416 (2017). 
54. Rouse, A. G., Williams, J. J., Wheeler, J. J. \& Moran, D. W. Cortical adaptation to a chronic microelectrocorticographic brain computer interface. J. Neurosci. 33, 1326-1330 (2013).

55. McCrimmon, C. M. et al. Electrocorticographic encoding of human gait in the leg primary motor cortex. Cereb. Cortex 28, 2752-2762 (2018).

56. Fitzsimmons, N. A., Lebedev, M. A., Peikon, I. D. \& Nicolelis, M. A. Extracting kinematic parameters for monkey bipedal walking from cortical neuronal ensemble activity. Front. Integr. Neurosci. 3,3 (2009).

57. Ma, X. et al. Decoding lower limb muscle activity and kinematics from cortical neural spike trains during monkey performing stand and squat movements. Front. Neurosci. 11, 44 (2017).

58. Waldert, S. et al. A review on directional information in neural signals for brain-machine interfaces. J. Physiol. Paris 103, 244-254 (2009).

59. Yin, M. et al. Wireless neurosensor for full-spectrum electrophysiology recordings during free behavior. Neuron 84, 1170-1182 (2014).

60. Borton, D. A., Yin, M., Aceros, J. \& Nurmikko, A. An implantable wireless neural interface for recording cortical circuit dynamics in moving primates. J. Neural Eng. 10, 026010 (2013).

61. Foster, J. D. et al. A freely-moving monkey treadmill model. J. Neural Eng. 11, 046020 (2014).

62. Schwarz, D. A. et al. Chronic, wireless recordings of large-scale brain activity in freely moving rhesus monkeys. Nat. Methods 11, 670-676 (2014).

63. Suner, S., Fellows, M. R., Vargas-Irwin, C., Nakata, G. K. \& Donoghue, J. P. Reliability of signals from a chronically implanted, silicon-based electrode array in non-human primate primary motor cortex. IEEE Trans. Neural Syst. Rehabil. Eng. 13, 524-541 (2005).

64. Barrese, J. C. et al. Failure mode analysis of silicon-based intracortical microelectrode arrays in non-human primates. J. Neural Eng. 10, 066014 (2013).

65. Simeral, J. D., Kim, S. P., Black, M. J., Donoghue, J. P. \& Hochberg, L. R. Neural control of cursor trajectory and click by a human with tetraplegia 1000 days after implant of an intracortical microelectrode array. J. Neural Eng. 8, 025027 (2011).

66. Chestek, C. A. et al. Long-term stability of neural prosthetic control signals from silicon cortical arrays in rhesus macaque motor cortex. J. Neural Eng. 8, 045005 (2011).

67. Milekovic, T., Ball, T., Schulze-Bonhage, A., Aertsen, A. \& Mehring, C. Detection of error related neuronal responses recorded by electrocorticography in humans during continuous movements. PLoS ONE 8, e55235 (2013).

68. Flouty, O. E. et al. Intracranial somatosensory responses with direct spinal cord stimulation in anesthetized sheep. PLoS ONE 8, e56266 (2013).

69. Musienko, P., van den Brand, R., Maerzendorfer, O., Larmagnac, A. \& Courtine, G. Combinatory electrical and pharmacological neuroprosthetic interfaces to regain motor function after spinal cord injury. IEEE Trans. Biomed. Eng. 56, 2707-2711 (2009).

70. Moritz, C. T., Lucas, T. H., Perlmutter, S. I. \& Fetz, E. E. Forelimb movements and muscle responses evoked by microstimulation of cervical spinal cord in sedated monkeys. J. Neurophysiol. 97, 110-120 (2007).

71. Sunshine, M. D. et al. Cervical intraspinal microstimulation evokes robust forelimb movements before and after injury. J. Neural Eng. 10, 036001 (2013).

72. Zimmermann, J. B., Seki, K. \& Jackson, A. Reanimating the arm and hand with intraspinal microstimulation. J. Neural Eng. 8, 054001 (2011).

73. Calixto, R. \& Mushahwar, V. Understanding the mechanisms and sites of action of intraspinal microstimulation. in Proceedings of the 12th Annual Conference of the International Functional Electrical Stimulation Society.

74. Rattay, F. Analysis of models for external stimulation of axons. IEEE Trans. Biomed. Eng. 33, 974-977 (1986).

75. McIntyre, C. C. \& Grill, W. M. Extracellular stimulation of central neurons: influence of stimulus waveform and frequency on neuronal output. J. Neurophysiol. 88, 1592-1604 (2002).

76. Gaunt, R. A., Prochazka, A., Mushahwar, V. K., Guevremont, L. \& Ellaway, P. H. Intraspinal microstimulation excites multisegmental sensory afferents at lower stimulus levels than local $\alpha$ - motoneuron responses. J. Neurophysiol. 96, 2995-3005 (2006).

77. Iles, J. F. Central terminations of muscle afferents on motoneurones in the cat spinal cord. J. Physiol. 262, 91-117 (1976).

78. Brown, A. G. \& Fyffe, R. E. The morphology of group Ia afferent fibre collaterals in the spinal cord of the cat. J. Physiol. 274, 111-127 (1978).

79. Burke, R. E. \& Glenn, L. L. Horseradish peroxidase study of the spatial and electrotonic distribution of group Ia synapses on type-identified ankle extensor motoneurons in the cat. J. Comp. Neurol. 372, 465-485 (1996).

80. Minassian, K., Hofstoetter, U., Tansey, K. \& Mayr, W. Neuromodulation of lower limb motor control in restorative neurology. Clin. Neurol. Neurosurg. 114, 489-497 (2012).

81. Ethier, C., Oby, E. R., Bauman, M. J. \& Miller, L. E. Restoration of grasp following paralysis through braincontrolled stimulation of muscles. Nature 485, 368-371 (2012).

82. Bouton, C. E. et al. Restoring cortical control of functional movement in a human with quadripleaturgia. Nature 533, 247-250 (2016).

83. Ajiboye, A. B. et al. Restoration of reaching and grasping movements through brain-controlled muscle stimulation in a person with tetraplegia: a proof-of-concept demonstration. Lancet 389, 1821-1830 (2017).

84. Giat, Y., Mizrahi, J. \& Levy, M. A musculotendon model of the fatigue profiles of paralyzed quadriceps muscle under FES. IEEE Trans. Biomed. Eng. 40, 664-674 (1993). 
85. Popovic, M. R., Popovic, D. B. \& Keller, T. Neuroprostheses for grasping. Neurol. Res. 24, 443-452 (2002).

86. House, P. A., MacDonald, J. D., Tresco, P. A. \& Normann, R. A. Acute microelectrode array implantation into human neocortex: preliminary technique and histological considerations. Neurosurg. Focus 20, 1-4 (2006).

87. Gerasimenko, Y., Roy, R. R. \& Edgerton, V. R. Epidural stimulation: comparison of the spinal circuits that generate and control locomotion in rats, cats and humans. Exp. Neurol. 209, 417-425 (2008).

\section{Acknowledgements}

The illustration in Fig. 1 was created by C. Beach. N.W. is a participant in the Charite Clinical Scientist Program funded by the Charité-Universitätsmedizin Berlin and the Berlin Institute of Health. This work was supported by Medtronic, the European Community's Seventh Framework Programme (CP-IP 258654, NeuWALK), the International Paraplegic foundation (IRP), a Consolidator Grant from the European Research Council (ERC-2015-CoG HOW2WALKAGAIN 682999), the Wyss Center in Geneva, the Russian Science Foundation (RSF grant 14-15-00788, P.M.), a Wings for Life Fellowship to G.C., Marie Curie COFUND EPFL fellowships to F.B. W. and T.M., and a Morton Cure Paralysis Fund fellowship to T.M., as well as by the Swiss National Science Foundation, including a Bonus of Excellence (310030B_166674), the National Center of Competence in Research (NCCR) Robotics, the Sino-Swiss Science and Technology Cooperation (IZLCZ3_156331), the NanoTera.ch program (SpineRepair) and the Sinergia program (CRSII3_160696).

\section{Author contributions}

M.C., F.B.W., J.G., E.M.M., N.W., T.M. and G.C. developed the methods to control the stimulation and optimize the electrode placement. P.S., N.P., P.M., J.B. and G.C. developed the surgical procedures. M.C., F.B.W., J.G., E.M.M. and T.M. performed the experiments in monkeys. N.W., E.M.M., J.G. and M.C. performed the experiments in rats. E.B. and G.C. supervised the experiments and animal procedures. M.C., F.B.W., J.G., E.M.M., N.W. and T.M. analyzed the data. M.C., F.B.W. and J.G. created the figures. M.C., F.B.W. and G.C. wrote the manuscript.

\section{Competing interests}

G.C., M.C., E.M.M., N.W., T.M., F.B.W., J.G. and J.B. hold various patents related to the present work. E.B. reports receipt of personal fees from Motac Neuroscience Ltd. UK and is a shareholder of Motac Holding, UK, and Plenitudes SARL, France. G.C. and J.B. are founders and shareholders of GTX Medical BV. The other authors declare no competing interests.

\section{Additional information}

Reprints and permissions information is available at www.nature.com/reprints.

Correspondence and requests for materials should be addressed to G.C.

Publisher's note: Springer Nature remains neutral with regard to jurisdictional claims in published maps and institutional affiliations.

Published online: 6 September 2018

\section{Related links}

Key references using this protocol

1. Wenger, N. et al. Nat. Med. 22, 138-145 (2016) https://doi.org/10.1038/nm.4025

2. Capogrosso, M. et al. Nature 539, 284-288 (2016) https://doi.org/10.1038/nature20118 